\title{
Modal Analysis of Substrate Integrated Waveguides With Rectangular Via-Holes Using Cavity and Multilayer Green's Functions
}

\author{
Malcolm Ng Mou Kehn, Senior Member, IEEE
}

\begin{abstract}
The substrate integrated waveguide (SIW) comprising rectangular via-holes is herein treated by a rigorous full-wave modal analysis using the moment method entailing Green's functions for rectangular cavities and planar multilayer structures in the spectral domain. Modal dispersion graphs generated by this solution approach are compared with those obtained by an independent commercial full-wave solver. The ability of the modal solution in treating SIW junctions and discontinuities is demonstrated by the treatment of an interconnection between a conventional waveguide and a SIW using the mode-matching technique. Inhomogeneities within SIWs can also be accommodated by the technique, as showcased by a specific example of the so-called hard sidewalled SIW. Three avenues of losses, namely, dielectric, side-leakage, and conductor losses, are also investigated, thereby elucidating a tradeoff between the latter two.
\end{abstract}

Index Terms-Green's function, moment method, spectral-domain technique, substrate integrated waveguide (SIW).

\section{INTRODUCTION}

$\mathbf{S}$ UBSTRATE integrated waveguides (SIWs) have received tremendous amounts of attention in recent years [1]-[3]. The chief advantages of these alternative structures include their ease and low cost of manufacture by virtue of economical through-hole techniques in printed-circuit technology, amenability to integration with planar circuits, high quality factor, high power-handling capacity, as well as lightweight nature, all these without compromising the overall essential properties of conventional solid-walled waveguides, which tend to be heavy, bulky, expensive to fabricate, and nonintegrable. The latter shortcoming is of particular importance to the power budget of millimeter-wave and terahertz systems since additional inherently lossy physical constructs are needed to interface ordinary waveguides with other stages in the system, tolling on the noise performance. Moreover, it is imperative that the various devices and components are not to be separately designed and handled. The ultimate solution to these issues is the full-scale integration and co-design of all components,

Manuscript received April 29, 2014; revised July 17, 2014; accepted July 19 2014. Date of publication August 08, 2014; date of current version October 02, 2014. This work was supported by the Ministry of Science and Technology, Taiwan, under Grant 102-2221-E-009-027.

The author is with the Department of Electrical Engineering, National Chiao Tung University, Hsinchu 30010, Taiwan (e-mail: malcolm.ng@ieee.org).

Color versions of one or more of the figures in this paper are available online at http://ieeexplore.ieee.org.

Digital Object Identifier 10.1109/TMTT.2014.2344626 devices, and circuits, active and passive ones alike, within the same design platform sharing a common processing technique, which will reduce the cost of design and fabrication, as well as enhance the overall system performance. As such, SIWs have become attractive and popular amongst microwave and antenna engineers.

Microwave and millimeter-wave devices embracing SIW technology include power dividers [4], [5], filters [6], and circulators [7], [8]. SIW-based antenna applications also come in a wide assortment, such as SIW waveguide slot-array antennas [9], [10], SIW-fed parallel-plate slot array [11], SIW-horn antennas [12]-[16], and SIW-fed antipodal linearly tapered slot antenna (ALTSA) [17], [18], just to name a few.

Via-holes of circular cross section have been customary in the implementation of SIWs due to their ease and low cost of manufacture. However, in the advent of new fabrication techniques such as deep reactive-ion etching (DRIE), inductively coupled plasma etching (ICPE), and other modern laser technologies, via-holes of any shape can today be produced, e.g., [19]. Square or rectangular posts [20]-[22] constitute one geometry that has become increasingly popular and widespread. Besides, by a preexisting accurate conversion formula [23] relating the geometries of circular and square via-holes (as showcased later in Section IX), studies into both shapes of pins are interchangeable and thus equally relevant.

Sections III-VI present a full-wave modal analytical treatment of the SIW made of rectangular via-holes (schematized in Fig. 1) by the moment method entailing Green's functions for rectangular cavities [24], [25] as well as planar multilayer structures in the spectral domain, the underlying approach entailing the latter being similar to that in [26]. The concepts behind the numerical search for eigenmodal resonances entailing repeated solutions will also be described in Section VII. Computed results of modal dispersion diagrams are validated with those obtained from a commercial full-wave solver in Section VIII. To underscore the distinctive traits of the present technique, its ability in accommodating complex scenarios such as those involving SIW inhomogeneities as well as in the presence of junctions and discontinuities shall also be demonstrated - through the studies of the so-called hard sidewalled SIW in Section XI (described more in that section) and the connection of an ordinary solid-walled waveguide with an SIW using the mode-matching technique in Section X. Thereafter, three avenues of losses, namely, dielectric, side-leakage, and conductor losses, will be investigated in Sections XII-XIV, 


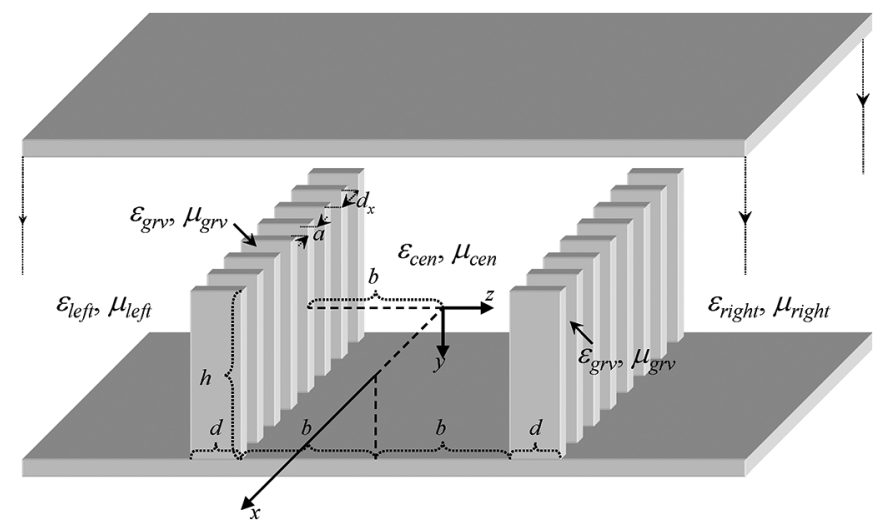

Fig. 1. Schematic and geometry of SIW.

respectively, from which a tradeoff between the latter two is elucidated This consequently paves the way for the explorations in Section XV of a modified form of SIW made of strip-grated sidewalls with enhanced benefits, thereby further substantiating the usefulness and relevance of the herein treatment of rectangular-shaped posts.

\section{Prologue to the Theory and Formulation}

It has been shown in [26] how the sidewall dipole-FSS loaded rectangular waveguide can be treated using the spectral multilayer Green's function by solving a five-layer infinitely planar structure with periodic virtual perfect electric conducting (PEC) walls perpendicular to the surface of the multilayer structure incorporated to simulate the metal waveguide walls perpendicular to the dielectric slab surfaces of the FSS. The same concept used there reapplies here for the treatment of the SIW.

The upcoming formulation spanning Sections III-VI adopts the route of full generality whereby all subregions of the SIW structure assume a different set of physical dimensions and material parameters, which is a healthy practice towards flexibility and universality that facilitate future modifications, and thus, further research. Nonetheless, as many SIWs do possess geometric central symmetry, simplification of the method is possible by the judicious use of magnetic and electric surfaces over the symmetry plane for odd $\mathrm{TE}_{(2 m+1), 0}$ and even $\mathrm{TE}_{2 m, 0}$ modes, respectively, reducing the size of the problem by half. By the use of symmetries (if applicable), the complexity of the formulation as well as the computing time will be reduced.

\section{Equivalent Magnetic Sources and Radiated FieldS}

The theory and formulation commence herein with the establishment of the current sources and their radiated fields throughout the various regions comprising the SIW structure.

\section{A. PEC-Equivalent Cavity-Aperture Magnetic Current}

The geometry of the SIW relevant to the analysis is shown in Fig. 1. The width and height along $z$ and $y$ of the central waveguiding region is $2 b$ and $h$, respectively. The dimensions of the rectangular pins along the width and axial $(z$ and $x)$ coordinate directions are $d$ and $d_{x}-a$, respectively, where $d_{x}$ is the unit cell-size (or period) of the pins array, and $a$ is the gap width of the aperture. The permittivities $\varepsilon$ and permeabilities $\mu$ of the center, left, right, and groove regions are attached with the respective subscripts cen, left, right, and grv.

The total transverse $x y$ component of the electric field observed over any one of the four scattering cavity apertures at $z=-b-d,-b, b$, or $b+d$ is then written as

$\vec{e}_{t}^{\mathrm{caav}}\left(x_{o}, y_{o}\right)=\sum_{p q r=1}^{N_{\mathrm{cav}}} A_{p q r} \vec{e}_{t_{p q r}}^{\mathrm{cav}}\left(x_{o}, y_{o}\right) \quad \vec{e}_{t_{p q r}}^{\mathrm{cav}}=\hat{x} e_{x_{p q r}}^{\mathrm{cav}}+\hat{y} e_{y_{p q r}}^{\mathrm{caav}}$

in which $e_{t}$ symbolizes the transverse ( $x$ and $y$ components only) electric vector modal field function of the rectangular waveguide having the same $a \times h$ cross section along $x$ and $y$ as the cavity apertures, and where $p q r$ signifies the modal triple-index, with $p$ and $q$ being integers associated with $p \pi / a$ and $q \pi / h$ and $r$ may denote TE or TM. The total number of waveguide modes considered is symbolized as $N_{\text {cav }}$. Clearly, $A_{p q r}$ represents the modal amplitude coefficient, being thus far unknown and to be solved for. Subsequently, the PEC-equivalent magnetic currents over the cavity apertures are given by

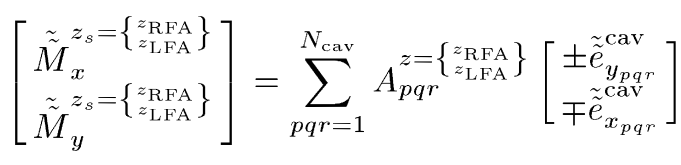

where $z_{\mathrm{RFA}}$ may assume $-b$ or $b+d$, with the RFA subscript denoting the right facing aperture, and $z_{\mathrm{LFA}}$ may take on $-b-d$ or $+b$, with the LFA subscript denoting the left facing aperture. These $z_{\mathrm{RFA}}$ and $z_{\mathrm{LFA}}$ in curly braces correspond to the upper and lower signs of the \pm symbol prefixed to the $e$-field functions on the right-hand side of the equation. These waveguide modal transverse electric field functions must be in their orthonormalized forms, as required by the dyadic Green's functions, and their explicit expressions are provided in Appendix A. The Fourier transforms of the magnetic currents in (2) into the spectral $\left(k_{x}, k_{y}\right)$ domain are stated as

$$
\begin{aligned}
& {\left[\begin{array}{l}
\tilde{\tilde{M}}_{x}^{z_{s}=\left\{\begin{array}{l}
z_{\mathrm{RFA}} \\
{ }_{\mathrm{RFFA}}
\end{array}\right\}}\left(k_{x}, k_{y}\right) \\
\tilde{\tilde{M}}_{y}^{z_{s}=\left\{\begin{array}{l}
z_{\mathrm{RFA}} \\
z_{\mathrm{LFA}}
\end{array}\right\}}\left(k_{x}, k_{y}\right)
\end{array}\right]}
\end{aligned}
$$

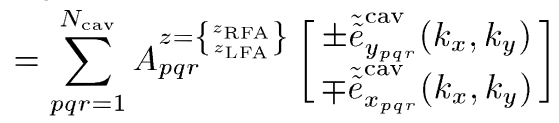

$$
\begin{aligned}
& \tilde{\tilde{e}}_{w_{p q r}}^{\mathrm{cav}}\left(k_{x}, k_{y}\right) \\
& =\int_{y=-\frac{h}{2}}^{y=\frac{h}{2}} \int_{x=-\frac{a}{2}}^{x=\frac{a}{2}} e_{w_{p q r}}^{\mathrm{cav}}(x, y) e^{j\left(k_{x} x+k_{y} y\right)} d x d y
\end{aligned}
$$

where $w$ may be $x$ or $y$. 


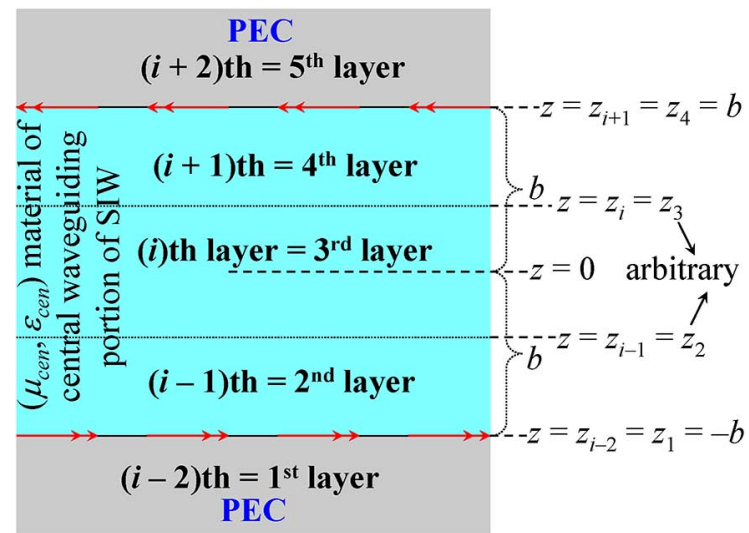

Fig. 2. Five-layer configuration for central waveguiding portion of the SIW used in core routine of spectral multilayer Green's function.

\section{B. Fields Radiated by PEC-Equivalent Cavity-Aperture Magnetic Currents into Various Regions}

Three regions into which the equivalent magnetic currents radiate fields are identified: the central waveguiding region and the two semi-infinite side regions.

1) Central Waveguiding Portion: Where a homogeneously filled central waveguiding portion of the SIW is concerned, the pertinent multilayer configuration for treatment by the core routine of the numerical spectral-domain multilayer Green's function (details of which are in Appendix B) would be a three-layer scenario comprising a central layer made of the material constituting the waveguiding portion, and which is sandwiched by two semi-infinite PEC layers that extend to positive and negative infinity along $z$. However, in anticipation of the subsequent treatment of the dielectric sidewall loaded hard SIW [27] later in Section XI, we shall instead consider a five-layer configuration shown in Fig. 2. The central third layer is labeled as the $i$ th layer, with the other layer indices with respect to this $i=3$, e.g., the first layer is the $(i-2)$ th layer while the topmost fifth layer is labeled as the $(i+2)$ th layer. The symbol $z_{i}$ represents the $z$-level of the upper boundary of the $i$ th layer.

The scattered fields within this central portion are triggered by two contributing spectral current sources, being the Fouriertransformed PEC-equivalent magnetic cavity-aperture currents laid out in Section III-A: 1 ) at the lower boundary $z=z_{i-2}=$ $z_{1}=-b$ and 2) at the upper boundary $z=z_{i+1}=z_{4}=+b$.

The following $(p q r)$ th modal basis functions for expanding the magnetic currents existing over the right-facing apertures and their Fourier-transformed spectral forms are first defined:

$$
\begin{aligned}
m_{\left\{\begin{array}{l}
x \\
y
\end{array}\right\}_{p q r}}(x, y) & = \pm e_{\substack{\left\{\begin{array}{c}
x \\
y
\end{array}\right\}_{p q r} \\
\text { cav }}}(x, y) ; \tilde{\tilde{m}}_{\left\{\begin{array}{c}
x \\
y
\end{array}\right\}_{p q r, m n}} \\
& = \pm \tilde{\tilde{e}}_{\left\{\begin{array}{c}
x \\
y \\
y
\end{array}\right\}_{p q r}}\left(k_{x_{m}}, k_{y_{m}}\right)
\end{aligned}
$$

in which the upper and lower signs of \pm correspond to the upper and lower symbols within the curly braces. By virtue of the discrete spectrum of the presently considered periodicity with periods along $x$ and $y$ being $d_{x}$ and $d_{y}=2 h$, these above Fourier-transformed modal basis current functions are evaluated at discrete values of $k_{x}$ and $k_{y}$ governed by

$$
k_{x_{m}}=k_{x_{m=0}}+2 m \pi / d_{x} \text { and } k_{y_{n}}=k_{y_{n=0}}+2 n \pi / d_{y}
$$

with $m$ and $n$ being integers, and

$k_{x_{m=0}}=k_{i} \sin \theta_{0} \cos \phi_{0}$ and $k_{y_{n=0}}=k_{i} \sin \theta_{0} \sin \phi_{0}=q \pi / h$

such that $k_{y_{n}}=(q+n) \pi / h$, and where $k_{i}=2 \pi f \sqrt{\mu_{i} \varepsilon_{i}}$. The angles $\theta_{0}$ and $\phi_{0}$ are measured from the $z$ - and $x$-axes, respectively, and they define the direction of the $(0,0)$ th dominant Floquet modal plane wave whose wavenumbers along $x$ and $y$ are $k_{x_{m=0}}$ and $k_{y_{n=0}}$, constituting the forcing wavenumbers along $x$ and $y$, respectively.

Using (B8), the spatial-domain fields in the $\ell$ th layer $(\ell=$ 2,3 , or 4 ) of Fig. 2 are represented by

$$
\begin{aligned}
& F_{w_{M_{x+y}}^{z= \pm b}}^{(\ell) \operatorname{cen}}(x, y, z)=F_{w_{M_{x+y}}^{\prime(\ell) \operatorname{cen}}}^{\prime= \pm}(z) e^{-j\left(k_{x_{m}} x+k_{y_{n}} y\right)}
\end{aligned}
$$

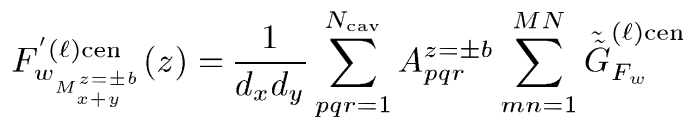

$$
\begin{aligned}
& \text { - }\left(\tilde{\tilde{m}}_{x_{p q r, m n}^{\mathrm{ex}}}^{z_{i \pm 1}^{\mathrm{x}}= \pm b}+\tilde{\tilde{m}}_{y_{p q r, m n}^{\mathrm{ex}}}^{z_{i \pm 1}^{\mathrm{e}}= \pm b}\right) \\
& \tilde{\tilde{m}}_{\left\{\begin{array}{c}
x \\
y \\
y
\end{array}\right\}_{p q r, m n}}^{z_{i \pm 1}^{\text {x }}= \pm b}=\mp \tilde{\tilde{m}}_{\left\{\begin{array}{l}
x \\
y
\end{array}\right\}_{p q r, m n}}
\end{aligned}
$$

noting that the exponential term of (8a) with $m$ and $n$ indices is within the summation over the Floquet modal harmonic index $m n$ in (8b). The field symbol $F$ is $E$ or $H, w$ is $x, y$, or $z$, and $\tilde{\tilde{G}}$ is the spectral multilayer Green's function of (B8), detailed in Appendix B. Notice that a cen superscript has been added to signify that the five-layer configuration of Fig. 2 for the central waveguiding portion is used in the core routine with top and bottom layers made of the PEC. Also note the distinction between indices $\ell$ and $i$, respectively, for the index of the layer (in Fig. 2) in which the field $F$ is observed and that of the one within which the $(m n)$ th spectral $(p q r)$ th basis magnetic current source $\tilde{\tilde{m}}_{w_{p q r}, m n}$ is located.

2) Terminal Left Region: $-\infty<z<-b-d$ : For the left semi-infinite terminal side region, where a single homogeneous medium is concerned, the pertinent multilayer configuration of the core routine of the numerical spectral-domain multilayer Green's function (details of which are in Appendix B) is simply a two-layer scenario with an interface at $z=-b-d$ comprising a PEC upper semi-infinite half-space layer that extends up to $z=+\infty$ (i.e., spans from $z=-b-d$ to $z=+\infty$ ) and a lower likewise semi-infinite half-space layer made of the material constituting the left semi-infinite terminal side region of the SIW that stretches down to $z=-\infty$ (i.e., exists from $z=-\infty$ to $z=-b-d$ ). However, readopting the same five-layer configuration of Fig. 2 for the central waveguiding portion for the liberty of stratifying the left-side region with four different materials, the structure of Fig. 3(a) shall be treated in the core routine. The same layer indexing as in Fig. 2 is reused.

This time, unlike the central waveguiding portion, the fields within this left-side terminal portion are due to current sources over just one boundary interface, being the PEC-equivalent magnetic cavity-aperture currents over the upper boundary at $z=z_{i+1}=z_{4}=-b-d$ just within the $(i+1)$ th $=4$ th 


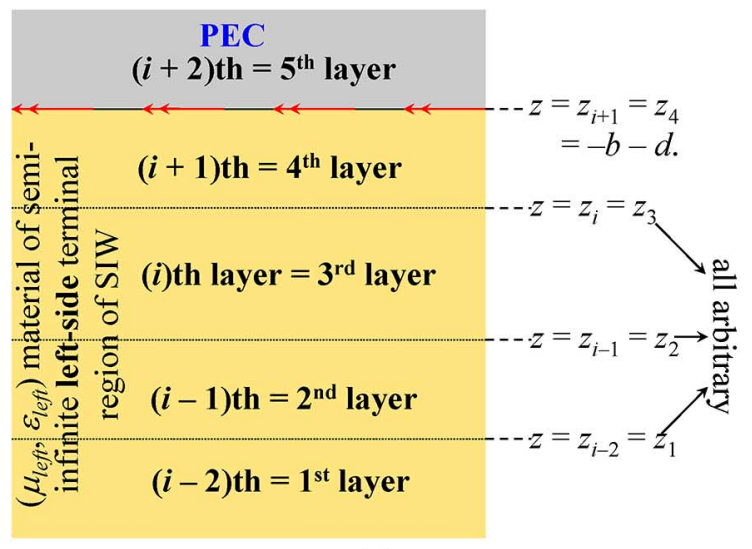

(a)

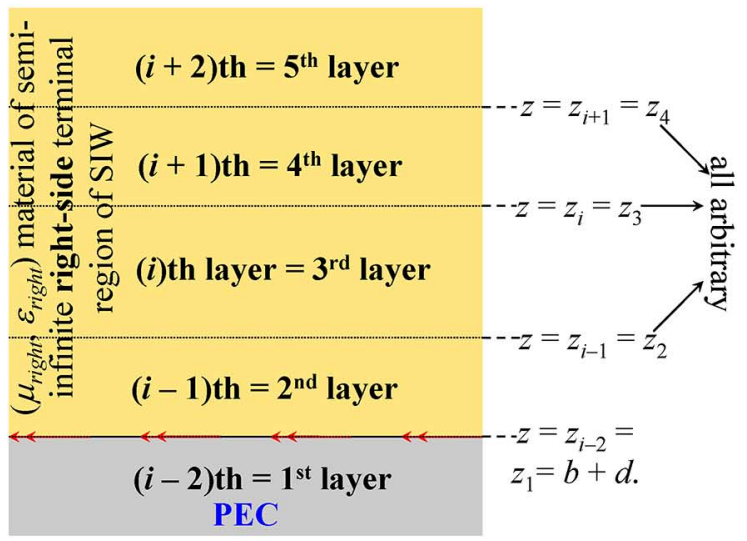

(b)

Fig. 3. (a) Five-layer configuration for the left semi-infinite terminal region of the SIW used in the core routine of the spectral multilayer Green's function. (b) Five-layer configuration for the right semi-infinite terminal region of the SIW used in the core routine of the spectral multilayer Green's function.

layer of Fig. 3(a). Again with (B8), the fields in the $\ell$ th layer $(\ell=1,2,3$, or 4$)$ of Fig. 3(a) are given by

$$
\begin{aligned}
& F_{w_{M_{x+y}}^{z=-b-d}}^{(\ell) \text { left }}(x, y, z)=F_{w_{M_{x+y}}^{z=-b-d}}^{\prime(\ell) \text { left }}(z) e^{-j\left(k_{x_{m}} x+k_{y_{n}} y\right)}
\end{aligned}
$$

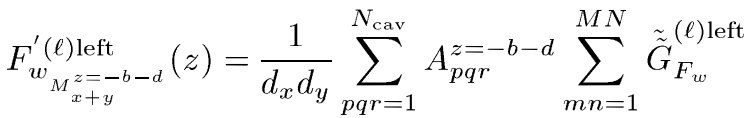

$$
\begin{aligned}
& \text { • }\left(\tilde{\tilde{m}}_{x_{p q r, m n}}^{z_{i_{1}=-b-d}^{\mathrm{ex}}}+\tilde{\tilde{m}}_{y_{p q r, m n}}^{z_{i+1}^{\mathrm{ex}}=-b-d}\right)
\end{aligned}
$$

$$
\tilde{\tilde{m}}_{\left\{\begin{array}{l}
x \\
y
\end{array}\right\}_{p q r, m n}}^{z_{i+1}^{\text {x }}=-b-d}=-\tilde{\tilde{m}}_{\left\{\begin{array}{l}
x \\
y
\end{array}\right\}_{p q r, m n}}
$$

with the same descriptions of the various symbols as for (8a) and the $m n$-indexed exponential term of (10a) is subjected to the summation over $m n$ in (10b). As before, notice this time, the left superscript attached to the Green's function to symbolize that the five-layer framework of Fig. 3(a) for the left region is used in the core routine with only the top layer being composed of the PEC. It is important to note that although the same symbol $i$ has been used in (8) and (10), the entities associated with it in both equations are different from one another by the reason that they pertain to two disparate multilayer configurations of Figs. 2 and 3(a), respectively.
3) Terminal Right Region: $b+d<z<\infty$ : As was for the left-side terminal region, the pertinent multilayer configuration of the spectral multilayer core routine for a homogeneous right-side terminal region is also simply a two-layer scenario, this time with the interface at $z=b+d$ comprising a lower semi-infinite half-space PEC layer that reaches down to $z=-$ infty (i.e., spans from $z=-\infty$ to $z=b+d$ ) and an upper likewise semi-infinite half-space layer made of the material constituting the right semi-infinite terminal side region of the SIW that extends up to $z=+\infty$ (i.e., exists from $z=b+d$ to $z=+\infty)$. However, retaining the same liberty of stratifying the right-side region with four different media as well, the five-layer configuration of Fig. 3(b) is applied to the core routine of the spectral multilayer Green's function.

The PEC-equivalent magnetic cavity-aperture currents responsible for driving the core routine are now located on the lower boundary at $z=z_{i-2}=z_{1}=b+d$, just within the $(i-1)$ th $=2$ nd layer. Once more by (B8), the fields in the $\ell$ th layer $(\ell=2,3,4$, or 5$)$ of Fig. 3(b) are given by

$$
\begin{aligned}
& F_{w_{M^{\prime}}^{z=b+d}}^{(\ell) \text { right }}(x, y, z)=F_{w_{M^{\prime}}}^{\prime(\ell) \text { right }}(z) e^{-j\left(k_{x_{m}} x+k_{y_{n}} y\right)} \\
& F_{w_{M}}^{\prime(\ell) \text { right }}(z)=\frac{1}{d_{x} d_{y}} \sum_{p q r=1}^{N_{\text {cav }}} A_{p q r}^{z=b+d} \sum_{m n=1}^{M N} \tilde{\tilde{G}}_{F_{w}}^{(\ell) \text { right }} \\
& \text { - }\left(\tilde{\tilde{m}}_{x_{p q r}, m n}^{z_{i_{1}}^{\mathrm{ex}}=b+d}+\tilde{\tilde{m}}_{y_{p q r, m n}}^{z_{i+1}^{\mathrm{ex}}=b+d}\right) \\
& \tilde{\tilde{m}}_{\left\{\begin{array}{l}
x \\
y
\end{array}\right\}_{p q r, m n}}^{z_{i-1}^{\mathrm{ex}}=b+d}=-\tilde{\tilde{m}}_{\left\{\begin{array}{c}
x \\
y
\end{array}\right\}_{p q r, m n}} .
\end{aligned}
$$

\section{Continuity of Tangential Magnetic Field COMPONENT ACROSS CAVITY APERTURES}

Through the sign cases of (2), depending on whether the PEC-equivalent cavity-aperture magnetic current is right or left facing, i.e., with the unit normal pointing towards the region into which fields are radiated being $+\hat{z}$ or $-\hat{z}$, the continuities of the tangential electric field components across the various cavity apertures are already implicitly satisfied. What remains is to enforce the continuity of the tangential magnetic field component.

\section{A. Inner Apertures at $z= \pm b$}

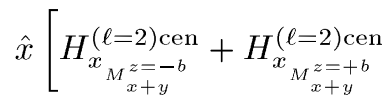

$$
\begin{aligned}
& \left.+\tilde{\tilde{G}}_{H_{x}}^{(\ell=2) \mathrm{cen}} \bullet \Psi_{\sigma=i}^{z_{\tau=i}^{\mathrm{ex} 1}{ }^{\circ}} e^{-j\left(k_{x_{0}} x+k_{y_{0}} y\right)}\right]_{z=-b} \\
& +\hat{y}\left[H_{y_{\substack{M_{x+y}^{z=-b} \\
x+y}}^{(\ell=2) \mathrm{cen}}}^{\left(\ell-H_{M_{x+y}}^{z=+b}\right.}\right. \\
& \left.+\tilde{\tilde{G}}_{H_{y}}^{(\ell=2) \mathrm{cen}} \bullet \Psi_{\sigma=i}^{z_{\tau=i}^{\mathrm{ex} 1}{ }^{\circ}} e^{-j\left(k_{x_{0}} x+k_{y_{0}} y\right)}\right]_{z=-b} \\
& =\sum_{p q r=1}^{N_{\mathrm{cav}}}\left[A_{p q r}^{z=-b-d} \operatorname{csch}\left(\gamma_{z_{p q r}}^{\mathrm{cav}} d\right)\right. \\
& \left.-A_{p q r}^{z=-b} \operatorname{coth}\left(\gamma_{z_{p q r}}^{\mathrm{cav}} d\right)\right] \vec{h}_{t_{p q r}}^{\mathrm{cav}}(x, y ; z=-b)
\end{aligned}
$$




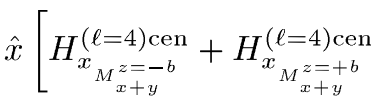

$$
\begin{aligned}
& \left.+\tilde{\tilde{G}}_{H_{x}}^{(\ell=4) \operatorname{cen}} \bullet \Psi_{\sigma=i}^{z_{\tau=i}^{\mathrm{ex} 1^{\circ}}} e^{-j\left(k_{x_{0}} x+k_{y_{0}} y\right)}\right]_{z=b} \\
& +\hat{y}\left[H_{y_{M_{x+y}^{z=-b}}}^{(\ell=4) \text { cen }}+H_{y_{M_{x+y}^{z=+b}}}^{(\ell=4) \text { cen }}\right. \\
& \left.+\tilde{\tilde{G}}_{H_{y}}^{(\ell=4) \operatorname{cen}} \bullet \Psi_{\sigma=i}^{z_{\tau=i}^{\mathrm{ex} 1}{ }^{\circ}} e^{-j\left(k_{x_{0}} x+k_{y_{0}} y\right)}\right]_{z=b} \\
& =\sum_{p q r=1}^{N_{\mathrm{cav}}}\left[A_{p q r}^{z=+b} \operatorname{coth}\left(\gamma_{z_{p q r}}^{\mathrm{cav}} d\right)\right. \\
& \left.-A_{p q r}^{z=b+d} \operatorname{csch}\left(\gamma_{z_{p q r}}^{\mathrm{cav}} d\right)\right] \vec{h}_{t_{p q r}}^{\mathrm{cav}}(x, y ; z=b)
\end{aligned}
$$

with the $(x, y)$ observation coordinates (albeit not explicit in the first two terms within parentheses on the left-hand sides of these equations) being over the left-side cavity aperture at $z=-b$ for (14) and over the right-side aperture at $z=b$ for (15), both with $-a / 2<x<a / 2$ and $-h / 2<y<h / 2 . \Psi_{\sigma}^{z_{\tau=i}^{\mathrm{ex} 0^{\circ}}}$ symbolizes the primary excitation source that is assumed to be located at $z=z_{\tau=i}^{\text {ex } 1^{\circ}}$ within the $\tau$ th $=i$ th $=3$ rd layer (middle part) of Fig. 2 for the central waveguiding portion. As before, $\Psi$ is $J$ or $M$ and $\sigma$ may be $x, y$, or $z$. The subscript $z=-b$ or $z=$ $b$ attached to the square brackets signify that the $H$-fields are observed at those $z$-levels. The right-hand sides of both these boundary-condition equations are acquired by the dyadic cavity Green's functions.

B. Outer Apertures at $z= \pm(b+d)$

$$
\begin{aligned}
& \hat{x}\left[H_{x_{M_{x+y}^{z=-b-d}}^{(\ell=4) \text { left }}}^{\left(\begin{array}{l}
x+y \\
z=-b-d
\end{array}\right.}\right]+\hat{y}\left[H_{y_{M_{x+y}^{z=-b}}^{(\ell=4) \text { left }}}\right]_{z=-b-d} \\
& =\sum_{p q r=1}^{N_{\text {cav }}}\left[A_{p q r}^{z=-b-d} \operatorname{coth}\left(\gamma_{z_{p q r}}^{\text {cav }} d\right)\right. \\
& \left.-A_{p q r}^{z=-b} \operatorname{csch}\left(\gamma_{z_{p q r}}^{\mathrm{cav}} d\right)\right] \vec{h}_{t_{p q r}}^{\mathrm{cav}}(x, y ; z=-b-d)
\end{aligned}
$$

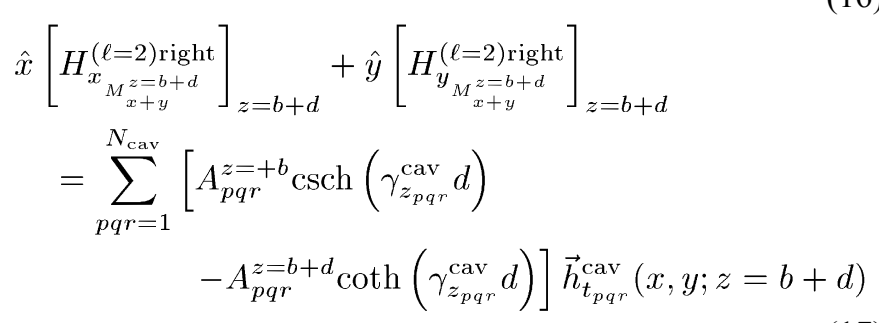

with the $(x, y)$ observation coordinates being over the left-end aperture of the left-side cavity at $z=-b-d$ for (16) and over the right-end aperture of the right-side cavity at $z=b+d$ for (17). As before, the functional dependencies (arguments) on the $(x, y)$ observation coordinates of the $H_{w}$ fields on the left-hand sides of both these equations are not explicitly shown.

\section{Galerkin Weighting: Generate System of Equations}

Taking $\int_{y=-h / 2} \int_{x=-a / 2} e_{t_{r s t}}^{\text {cav }}(x, y) \times_{---} \cdot \hat{z} d x d y$ throughout (14)-(17), each for $r s t=1,2, \ldots, N_{\text {cav }}$, the following four equations are, respectively, obtained:

$$
\begin{aligned}
& {\left[H_{y_{M_{x+y}^{z=-b}}^{(\ell=2) \mathrm{cen}}+H_{y_{M_{x+y}^{z=+b}}^{\prime(\ell=2) \mathrm{cen}}}^{\left({ }^{z}=-b\right.}}\right]_{z=-b} \tilde{\tilde{e}}_{x_{r s t} \mathrm{cav}}\left(-k_{x_{m}},-k_{y_{n}}\right)}
\end{aligned}
$$

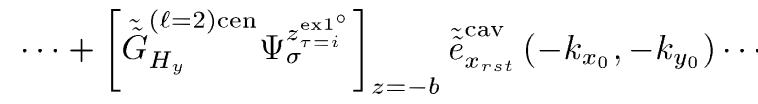

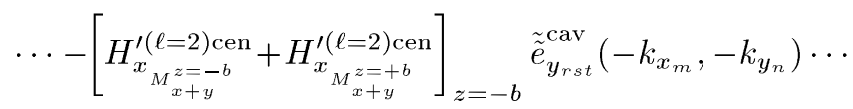

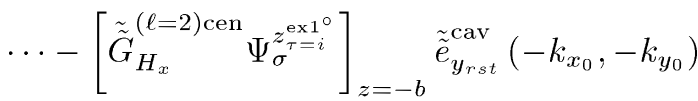

$$
\begin{aligned}
& =A_{r s t}^{z=-b-d} \operatorname{csch}\left(\gamma_{z_{r s t}}^{\mathrm{cav}} d\right)-A_{r s t}^{z=-b} \operatorname{coth}\left(\gamma_{z_{r s t}}^{\mathrm{cav}} d\right)
\end{aligned}
$$

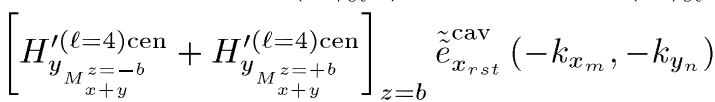

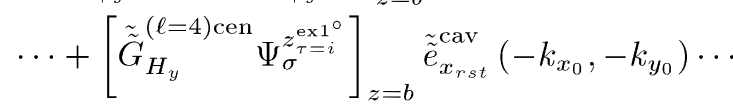

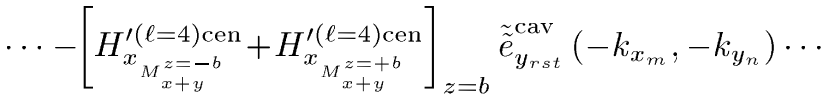

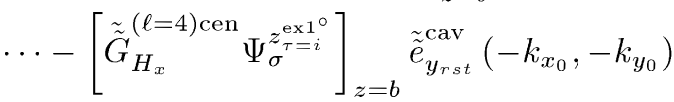

$$
\begin{aligned}
& =A_{r s t}^{z=+b} \operatorname{coth}\left(\gamma_{z_{r s t}}^{\mathrm{cav}} d\right)-A_{r s t}^{z=b+d} \operatorname{csch}\left(\gamma_{z_{r s t}}^{\mathrm{cav}} d\right) \\
& {\left[H_{y_{M_{x+y}^{z=-b-d}}^{\prime(\ell=4) \text { left }}}^{z=-b-d}\right]_{z=x_{r s t}^{\text {cav }}}\left(-k_{x_{m}},-k_{y_{n}}\right)}
\end{aligned}
$$

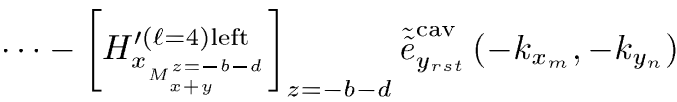

$$
\begin{aligned}
& =A_{r s t}^{z=-b-d} \operatorname{coth}\left(\gamma_{z_{r s t}}^{\text {cav }} d\right)-A_{r s t}^{z=-b} \operatorname{csch}\left(\gamma_{z_{r s t}}^{\text {cav }} d\right) \\
& {\left[H_{y_{M_{x+y}^{z}=b+d}^{\prime(\ell=2) \text { right }}}^{\left({ }_{x=b+d}\right.}\right]_{\tilde{e}_{x_{r s t}}^{\text {cav }}}\left(-k_{x_{m}},-k_{y_{n}}\right) \cdots} \\
& \cdots-\left[H_{x_{M_{x+y}^{z=b+d}}^{\prime(\ell=2) \text { right }}}^{\left(\ell+{ }^{2}\right.}\right]_{z=b+d} \tilde{\tilde{e}}_{y_{r s t}^{\mathrm{cav}}}\left(-k_{x_{m}},-k_{y_{n}}\right) \\
& =A_{r s t}^{z=+b} \operatorname{csch}\left(\gamma_{z_{r, s t}}^{\mathrm{cav}} d\right)-A_{r s t}^{z=b+d} \operatorname{coth}\left(\gamma_{z_{r} s t}^{\mathrm{cav}} d\right)
\end{aligned}
$$

whereby all right-hand sides are the result of waveguide mode orthonormality, as established in Appendix A. These generate a total of $4 N_{\text {cav }}$ equations sufficient for solving this same total number of unknown modal amplitude coefficients.

\section{CONSTRUCTION OF MATRIX EQUATION}

Anticipating the sequence of the unknown amplitude modal coefficients as placed in the $4 N_{\text {cav }} \times 1$ column-vector of (22), shown at the bottom of this page, with the nonconjugate transpose operators, $\mathrm{T}$ merely for compactness and thus saving

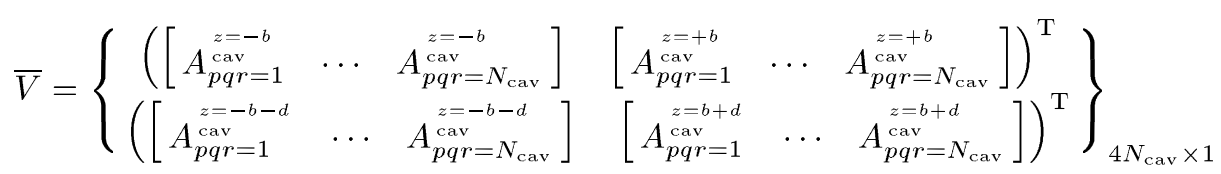


manuscript space (prevent taking up excessive space vertically downwards), the upcoming matrix operations are performed. Defining $\kappa=1 /\left(d_{x} d_{y}\right)$, using underscores to signify that their associated quantities are matrices, parenthesized row and column index symbols explicitly indicating the structural forms of the matrices, and with $w$ being $x$ or $y$, the matrices shown at the bottom of this page are constructed such that

$$
\begin{aligned}
& {\left[M_{11}\right]_{N_{\text {cav }} \times N_{\text {cav }}}} \\
& =\left[\underline{\tilde{\tilde{e}}}_{x_{p q r}}^{*}(r s t, m n)\right]_{N_{\mathrm{cav}} \times M N}
\end{aligned}
$$

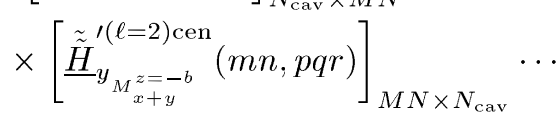

$$
\begin{aligned}
& \cdots-\left[\underline{\tilde{\tilde{e}}}_{y_{p q r}}^{*}(r s t, m n)\right]_{N_{\mathrm{cav}} \times M N}
\end{aligned}
$$

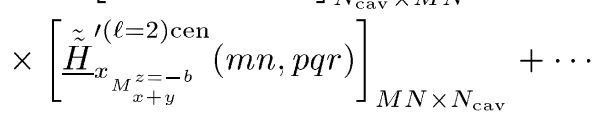

$$
\begin{aligned}
& \cdots+[\Upsilon(r s t, r s t)]_{N_{\text {cav }} \times N_{\text {cav }}} \\
& {\left[M_{12}\right]_{N_{\text {cav }} \times N_{\text {cav }}}} \\
& =\left[\underline{\tilde{\tilde{e}}}_{x_{p q r}}^{*}(r s t, m n)\right]_{N_{\mathrm{cav}} \times M N} \\
& \times\left[\underline{\tilde{\tilde{H}}}_{y_{\substack{x+y \\
z=+b}}^{(\ell=2) \mathrm{cen}}(m n, p q r)}(m]_{M N \times N_{\mathrm{cav}}} \cdots\right.
\end{aligned}
$$

$$
\begin{aligned}
& \cdots-\left[\underline{\tilde{\tilde{e}}}_{y_{p q r}}^{*}(r s t, m n)\right]_{N_{\mathrm{cav}} \times M N} \\
& \times\left[\underline{\tilde{\tilde{H}}}_{\substack{M_{x+y}^{z=+b} \\
(\ell=2) \mathrm{cen}}}(m n, p q r)\right]_{M N \times N_{\text {cav }}} \\
& {\left[M_{13}\right]_{N_{\text {cav }} \times N_{\text {cav }}}} \\
& =-[\mho(r s t, r s t)]_{N_{\text {cav }} \times N_{\text {cav }}} ; \\
& {\left[M_{14}\right]_{N_{\text {cav }} \times N_{\text {cav }}}} \\
& =[\text { zeros }]_{N_{\text {cav }} \times N_{\text {cav }}} \\
& {\left[M_{21}\right]_{N_{\text {cav }} \times N_{\text {cav }}}} \\
& =\left[\underline{\tilde{\tilde{e}}}_{x_{p q r}}^{*}(r s t, m n)\right]_{N_{\mathrm{cav}} \times M N} \\
& \times\left[\underline{\tilde{\tilde{H}}}_{y_{M_{x+y}^{z=-b}}^{\prime(\ell=4) \mathrm{cen}}(m n, p q r)}(m]_{M N \times N_{\mathrm{cav}}} \cdots\right. \\
& \cdots-\left[\underline{\tilde{\tilde{e}}}_{y_{p q r}}^{*}(r s t, m n)\right]_{N_{\mathrm{cav}} \times M N} \\
& \times\left[\underline{\tilde{\tilde{H}}}_{\left.M_{\substack{x+y \\
z=-b}}^{\prime(\ell=4) \mathrm{cen}}(m n, p q r)\right]_{M N \times N_{\text {cav }}}}\right. \\
& {\left[M_{22}\right]_{N_{\text {cav }} \times N_{\text {cav }}}} \\
& =\left[\underline{\tilde{\tilde{e}}}_{x_{p q r}}^{*}(r s t, m n)\right]_{N_{\mathrm{cav}} \times M N}
\end{aligned}
$$

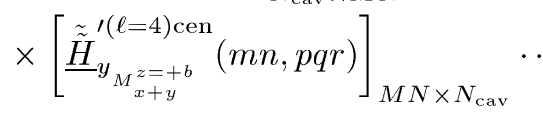

$$
\begin{aligned}
& {\left[\underline{\tilde{\tilde{H}}}_{w_{M_{x+y}^{z= \pm b}}}^{(\ell=2) \mathrm{cen}}(m n, p q r)\right]_{M N \times N_{\text {cav }}}} \\
& =\kappa\left[\tilde{\tilde{G}}_{H_{w}}^{(\ell=2) \operatorname{cen}} \bullet\left(\tilde{\tilde{m}}_{x_{p q r}, m n}^{z_{i \pm 1}^{\text {ex }}= \pm b}+\tilde{\tilde{m}}_{y_{p q r, m n}}^{z_{i \pm 1}^{\text {ex }}= \pm b}\right)\right]_{z=-b} \\
& {\left[\underline{\tilde{\tilde{H}}}_{w_{\substack{x+y \\
\text { zatb }}}^{\prime(\ell=4) \text { cen }}}^{(m n, p q r)}\right]_{M N \times N_{\text {cav }}}} \\
& =\kappa\left[\tilde{\tilde{G}}_{H_{w}}^{(\ell=4) \operatorname{cen}} \bullet\left(\tilde{\tilde{m}}_{x_{p q r, m n}}^{z_{i \pm 1}^{\text {ex }}= \pm b}+\tilde{\tilde{m}}_{y_{p q r, m n}}^{z_{i \pm 1}^{\text {ex }}= \pm b}\right)\right]_{z=b} \\
& {\left[\underline{\tilde{\tilde{H}}}_{w_{M+y}^{z=-b-d}}^{\prime(\ell=4) \text { left }}(m n, p q r)\right]_{M N \times N_{\text {cav }}}} \\
& =\kappa\left[\tilde{\tilde{G}}_{H_{w}}^{(\ell=4) \text { left }} \bullet\left(\tilde{\tilde{m}}_{x_{p q r}, m n}^{z_{i+1}^{\text {ex }}=-b-d}+\tilde{\tilde{m}}_{y_{p q r, m n}}^{z_{i+1}^{\text {ex }}=-b-d}\right)\right]_{z=-b-d} \\
& {\left[\underline{\tilde{\tilde{H}}}_{w}^{\prime(\ell=2) \text { right }}{ }_{\substack{z=b+d \\
x+y}}^{(m n, p q r)}\right]_{M N \times N_{\text {cav }}}} \\
& =\kappa\left[\tilde{\tilde{G}}_{H_{w}}^{(\ell=2) \text { right }} \bullet\left(\tilde{\tilde{m}}_{x_{p q r}, m n}^{z_{i-1}^{\text {ex }}=b+d}+\tilde{\tilde{m}}_{y_{p q r}, m n}^{z_{i-1}^{\text {ex }}=b+d}\right)\right]_{z=b+d} \\
& {\left[\underline{\tilde{\tilde{e}}}_{w_{r s t}}^{*}(r s t, m n)\right]_{N_{\text {cav }} \times M N}=\tilde{\tilde{e}}_{w_{p q r}}^{\mathrm{cav}_{p}}\left(-k_{x_{m}},-k_{y_{n}}\right)} \\
& {\left[\left\{\begin{array}{c}
\Upsilon \\
\mho
\end{array}\right\}(r s t, r s t)\right]}
\end{aligned}
$$

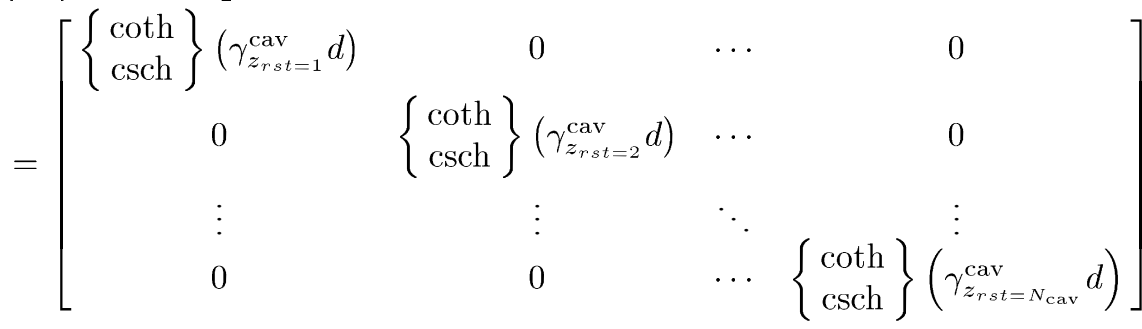




$$
\begin{aligned}
& \cdots-\left[\underline{\tilde{e}}_{y_{p q r}}^{*}(r s t, m n)\right]_{N_{\mathrm{cav}} \times M N} \\
& \times\left[\underline{\tilde{\tilde{H}}}_{M_{x+y}^{z=+b}}^{(\ell=4) \mathrm{cen}}(m n, p q r)\right]_{M N \times N_{\mathrm{cav}}} \cdots \\
& \cdots-[\Upsilon(r s t, r s t)]_{N_{\text {cav }} \times N_{\text {cav }}} \\
& {\left[M_{23}\right]_{N_{\text {cav }} \times N_{\text {cav }}}} \\
& =[\text { zeros }]_{N_{\text {cav }} \times N_{\text {cav }}} ; \\
& {\left[M_{24}\right]_{N_{\text {cav }} \times N_{\text {cav }}}} \\
& =[\mho(r s t, r s t)]_{N_{\mathrm{cav}} \times N_{\mathrm{cav}}} \\
& {\left[M_{31}\right]_{N_{\text {cav }} \times N_{\text {cav }}}} \\
& =[\mho(r s t, r s t)]_{N_{\text {cav }} \times N_{\text {cav }}} \\
& {\left[M_{32}\right]_{N_{\text {cav }} \times N_{\text {cav }}}} \\
& =\left[M_{34}\right]_{N_{\text {cav }} \times N_{\text {cav }}} \\
& =[\text { zeros }]_{N_{\text {cav }} \times N_{\text {cav }}} \\
& {\left[M_{33}\right]_{N_{\text {cav }} \times N_{\text {cav }}}} \\
& =\left[\underline{\tilde{\tilde{e}}}_{x_{p q r}}^{*}(r s t, m n)\right]_{N_{\mathrm{cav}} \times M N} \\
& \times\left[\underline{\tilde{\tilde{H}}}_{y_{x+y}^{z=-b-d}}^{\prime(\ell=4) \text { left }}(m n, p q r)\right]_{M N \times N_{\text {cav }}} \cdots \\
& \cdots-\left[\underline{\tilde{\tilde{e}}}_{y_{p q r}}^{*}(r s t, m n)\right]_{N_{\mathrm{cav}} \times M N}
\end{aligned}
$$

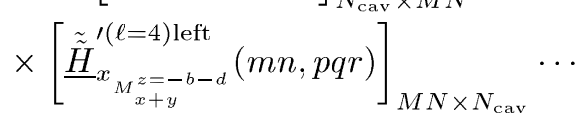

$$
\begin{aligned}
& \cdots-[\Upsilon(r s t, r s t)]_{N_{\text {cav }} \times N_{\text {cav }}} \\
& {\left[M_{41}\right]_{N_{\text {cav }} \times N_{\text {cav }}}} \\
& =\left[M_{43}\right]_{N_{\text {cav }} \times N_{\text {cav }}} \\
& =\operatorname{zeros}_{N_{\text {cav }} \times N_{\text {cav }}} \\
& {\left[M_{42}\right]_{N_{\text {cav }} \times N_{\text {cav }}}} \\
& =-[\mho(r s t, r s t)]_{N_{\text {cav }} \times N_{\text {cav }}} \\
& {\left[M_{44}\right]_{N_{\text {cav }} \times N_{\text {cav }}}} \\
& =\left[\underline{\tilde{\tilde{e}}}_{x_{p q r}}^{*}(r s t, m n)\right]_{N_{\mathrm{cav}} \times M N} \\
& \times\left[\underline{\tilde{\tilde{H}}}_{y_{M_{x+y}^{z=b+d}}^{(\ell=2) \mathrm{right}}}^{(m n, p q r)}\right]_{M N \times N_{\mathrm{cav}}} \ldots \\
& \cdots-\left[\underline{\tilde{\tilde{e}}}_{y_{p q r}}^{*}(r s t, m n)\right]_{N_{\mathrm{cav}} \times M N} \\
& \times\left[\underline{\tilde{\tilde{H}}}_{M_{x+y}^{z=b+d}}^{\prime(\ell=2) \mathrm{right}}(m n, p q r)\right]_{M N \times N_{\text {cav }}}+\cdots \\
& \cdots+[\Upsilon(r s t, r s t)]_{N_{\text {cav }} \times N_{\text {cav }}}
\end{aligned}
$$

$$
\begin{aligned}
& {\left[X^{\left(\left\{\begin{array}{l}
2 \\
4
\end{array}\right\}\right)}(r s t, 1)\right]_{N_{\text {cav }} \times 1}} \\
& =\left[\tilde{\tilde{G}}_{H_{y}}^{\left(\ell=\left\{\begin{array}{l}
2 \\
4
\end{array}\right\}\right) \mathrm{cen}} \bullet \Psi_{\sigma}^{z_{\tau=i}^{\mathrm{ex}}}\right]_{z=\mp b} \tilde{\tilde{e}}_{x_{r s t} \mathrm{cav}}^{\mathrm{cos}}\left(-k_{x_{0}},-k_{y_{0}}\right) \cdots \\
& \cdots-\left[\tilde{\tilde{G}}_{H_{x}}^{\left(\ell=\left\{\begin{array}{l}
2 \\
4
\end{array}\right\}\right) \mathrm{cen}} \bullet \Psi_{\sigma}^{z_{\tau=i}^{\mathrm{ex} 1}{ }^{\circ}}\right]_{z=\mp b} \tilde{\tilde{e}}_{y_{r s t} \mathrm{cav}}\left(-k_{x_{0}},-k_{y_{0}}\right) .
\end{aligned}
$$

Subsequently, the ultimate matrix equation is generated as the equation shown at the bottom of this page, where again the nonconjugate transpositions involved in the latter vector are just to avoid using up too much space vertically downward. This final matrix equation may then be solved for the modal amplitude coefficients in $\bar{V}$.

Solution of this latter inhomogeneous matrix equation with a nonzero vector $\bar{X}$ constitutes a physical driven-type problem involving the presence of an excitation source. However, within the class of eigenvalue problems [28], [29] without the presence of any source, the foregoing formulation remains fully effective by simply replacing $\bar{X}$ by a null vector. In order for nontrivial solutions, the determinant of the matrix $\bar{M}$ must vanish, the conditions of which are searched for numerically (as discussed in Section VII), thereby constituting the detected eigenmodal resonances. It is also mentioned that the present modal treatment approach shares the same aspect of considering just one unit cell as in eigenvalue problems.

\section{Detection of Eigenmodal Resonances}

This section describes the concepts behind the numerical search for eigenmodal resonances via repeated solutions of the SIW according to the formulations in Section VI.

Consider Fig. 4, in which the length of any test wavevector arrow is $k_{i}^{\text {test }}$. The angle which this vector makes with the $z$-axis is $\theta_{i}^{\text {test }}$, whereas the angle between the $x$-axis and the projection of the test wave-vector arrow onto the $x y$-plane is $\phi_{i}^{\text {test }}$.

Upon fixing $k_{x}^{\text {res }}$, with $k_{y_{n=0}}=q \pi / h$ fixed of course, and thus a correspondingly fixed $\phi_{i}^{\text {test }}=\phi_{i}^{\text {res }}$ (see the upcoming equations, especially the last one), then a $k_{i}^{\text {test }}$ that probably corresponds to the fixed $k_{x}^{\text {res }}$ is guessed, with a corresponding $\theta_{i}^{\text {test }}=\sin ^{-1}\left[(q \pi / h) /\left(k_{i}^{\text {test }} \sin \phi_{i}^{\text {res }}\right)\right]$. Subsequently, $k_{i}^{\text {test }}$, and thus, $\theta_{i}^{\text {test }}$ are varied over a range in which $k_{i}^{\text {test }}$ falls within. The SIW structure is then repeatedly solved by the formulation of Sections III-VI for the various $\left(k_{i}^{\text {test }}, \theta_{i}^{\text {test }}\right)$ cases until the resonance $k_{i}^{\text {test }}=k_{i}^{\text {res }}$ is found. That resonant

$$
\begin{aligned}
& \bar{M} \bar{V}=\bar{X} \\
& \bar{M}=\left\{\begin{array}{llll}
{\left[M_{11}\right]} & {\left[M_{12}\right]} & {\left[M_{13}\right]} & {\left[M_{14}\right]} \\
{\left[M_{21}\right]} & {\left[M_{22}\right]} & {\left[M_{23}\right]} & {\left[M_{24}\right]} \\
{\left[M_{31}\right]} & {\left[M_{32}\right]} & {\left[M_{33}\right]} & {\left[M_{34}\right]} \\
{\left[M_{41}\right]} & {\left[M_{42}\right]} & {\left[M_{43}\right]} & {\left[M_{44}\right]}
\end{array}\right\}_{4 N_{\text {cav }} \times 4 N_{\text {cav }}}
\end{aligned}
$$

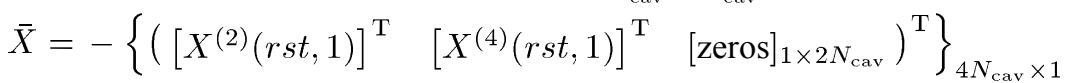




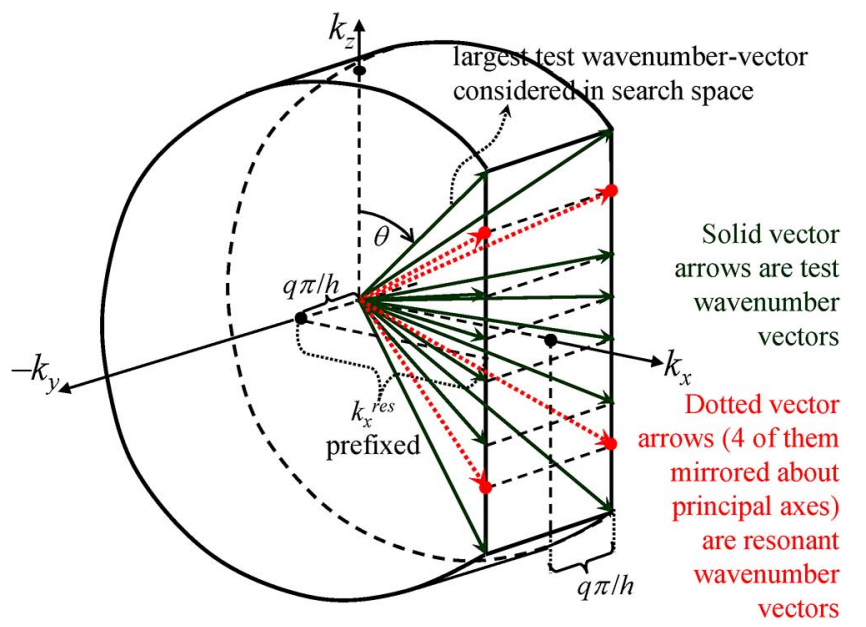

Fig. 4. Schematic of concept of resonance search. Arrows represent search space of wave-vectors. Original sphere of radius $k$ (so-called $k$-sphere) cut by three planes: two by the sides at $k_{y n=0}=\pi q \pi / h$ and one on the front at $k_{x}=k_{x}^{\text {res }}$. The cut sphere as shown here happens to illustrate the $k$-sphere of the largest test wavenumber vector considered in the shown search space (as indicated). There is no single fixed $k$-sphere-instead, the $k$-sphere radius is varied, i.e., frequency is varied.

frequency pertaining to the resonant $k_{i}$ is then the detected eigenmodal frequency.

$$
\begin{aligned}
& k_{i}^{\text {test }} \sin \theta_{i}^{\text {test }} \sin \phi_{i}^{\text {test }}=\frac{q \pi}{h} \\
& \text { fixed } \Rightarrow \theta_{i}^{\text {test }}=\sin ^{-1}\left(\frac{\frac{q \pi}{h}}{k_{i}^{\text {test }} \sin \phi_{i}^{\text {test }}}\right) \\
& k_{i}^{\text {test }} \sin \theta_{i}^{\text {test }} \cos \phi_{i}^{\text {test }}=k_{x}^{\text {res }}: \text { user-fixed. }
\end{aligned}
$$

Hence,

$$
\phi_{i}^{\text {test }}=\tan ^{-1}\left[\frac{\left(\frac{q \pi}{h}\right)}{k_{x}^{\text {res }}}\right]: \text { fixed. }
$$

This juncture marks the completion of the theory and formulation of the modal analysis. What follows next are sections that present numerical results and discussions associated with various types of studies. Without further specifications, the following declarations are made: 1) nonmagnetic materials are assumed throughout all computed cases, i.e., $\mu_{\mathrm{cen}}=\mu_{\text {left }}=$ $\mu_{\text {right }}=\mu_{0}$ and 2) the groove permittivity $\varepsilon_{\text {grv }}$ shall take on the same value as that of its surrounding slabs, specifically the central region $\varepsilon_{\text {cen }}$ and either side: $\varepsilon_{\text {left }}$ or $\varepsilon_{\text {right }}$.

\section{VALIDATION WITH COMMERCIAL SOLVER}

The modal characteristics of the SIW computed using the formulation of the preceding sections shall be compared with those simulated by the commercial full-wave software CST Microwave Studio. In particular, comparisons in terms of the dispersion diagram will be made. With reference to Fig. 1, the parameters of the SIW arbitrarily selected for showcasing the validation are first laid out: $b=5 \mathrm{~mm}, h=5 \mathrm{~mm}, d=0.2 b=$ $1 \mathrm{~mm}, d_{x}=0.8 d=0.8 \mathrm{~mm}, a=d_{x} / 2=0.4 \mathrm{~mm}, \mu_{\text {cen }}=\mu_{0}$, $\varepsilon_{\text {cen }}=2.2 \varepsilon_{0}, \mu_{\text {left }}=\mu_{\text {right }}=\mu_{0}$, and $\varepsilon_{\text {left }}=\varepsilon_{\text {right }}=\varepsilon_{0}$.

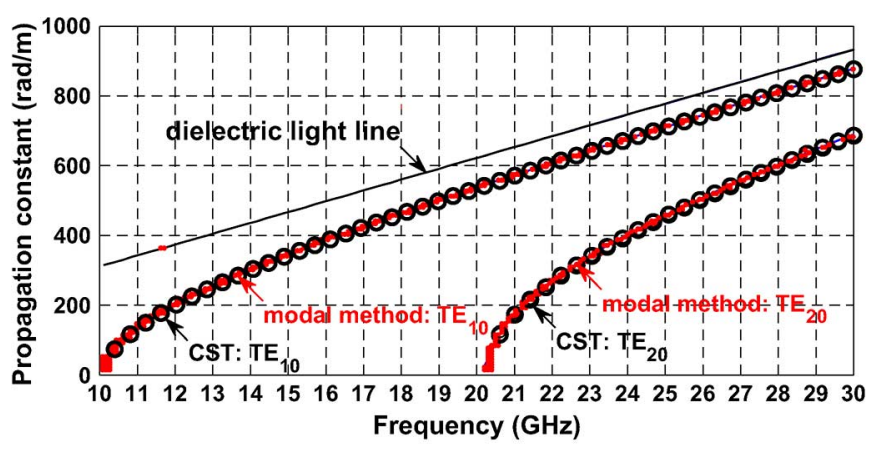

(a)

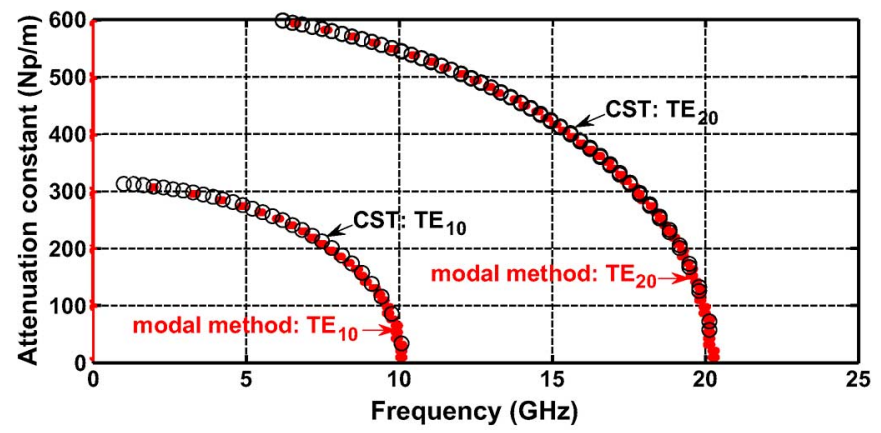

(b)

Fig. 5. Validation of present full-wave method with CST for dispersion diagram of: (a) propagating modes and (b) evanescent modes. For both plots, fine-dot and circle markers pertain to full-wave method and CST simulations, respectively.

The modal dispersion curves of the SIW generated using the herein presented full-wave solution approach are compared in Fig. 5(a) with those generated by CST. Evidently, excellent agreement between the two independent tools is observed for both the $\mathrm{TE}_{10}$ and $\mathrm{TE}_{20}$ modes of the present geometrical case. The variation of the attenuation constant with frequency for evanescent modes is also portrayed in Fig. 5(b). Once more, both tools are seen to be mutually corroborative.

The computation times of the present technique and the eigensolver of the CST software are also compared. The processing durations of both tools, executed on an Intel Core i7-3820 CPU at $3.60-\mathrm{GHz}$ clock speed and supported by $64.0 \mathrm{~GB}$ of RAM, were recorded for the following set of parameters: center width $2 b=10 \mathrm{~mm}$, height $h=1.5 \mathrm{~mm}$, $\mu_{\mathrm{cen}}=\mu_{\text {left }}=\mu_{\text {right }}=\mu_{0}$, and $\varepsilon_{\text {cen }}=\varepsilon_{\text {left }}=\varepsilon_{\text {right }}=\varepsilon_{0}$, period $d_{x}$ as a fraction of the width $2 b$, i.e., $d_{x} /(2 b)=0.4$, and square-pin diameter $d=t$ as a fraction of the period $d_{x}$, i.e., $t / d_{x}=0.4$. For the CST solver, a total computational time of $3 \mathrm{~h}, 12 \mathrm{~min}$, and $14 \mathrm{~s}$ to acquire numerical data at 180 frequencies was noted, whereas the herein proposed method took just $1482.633 \mathrm{~s}$ equivalent to $24 \mathrm{~min}$ and $42.633 \mathrm{~s}$ for the same number of frequency points. Therefore, the present approach is considerably more computationally efficient.

\section{Relation of Square Via-Holes With Circular Pins}

There are many published works in the literature that report the use of square or rectangular pins in SIWs. In [30] and [31], circular vias were translated to square ones that share the same cross-sectional areas. A more accurate, but still simple square-to-circular via-hole conversion was proposed in [23] 


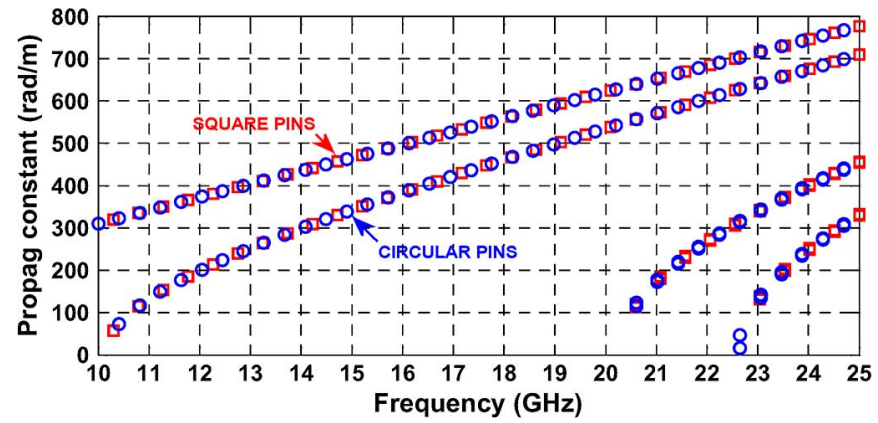

Fig. 6. CST-generated dispersion of SIW composed of square-pins (square markers) and the equivalent circular posts (circle markers) according to conversion (23). For square pins: $d=t=0.4 \mathrm{~mm}$; for circular pins, $d_{\text {circ }}=$ $2 t /(1+1 / \sqrt{ } 2)=0.46863 \mathrm{~mm}$. Width $2 b=10 \mathrm{~mm}$, height $h=5 \mathrm{~mm}$, period $d_{x}=0.8 \mathrm{~mm}$, and dielectric slab $\varepsilon_{\mathrm{rel}}=2.2$ throughout.

and used in numerous places [32], [33], which entails merely the arithmetic mean of the inscribed and circumscribed squares of the circular via

$$
a_{\mathrm{sq}}=\frac{d_{\mathrm{circ}}}{2}\left(1+\frac{1}{\sqrt{2}}\right)
$$

for which a circular via of diameter $d_{\text {circ }}$ may be well emulated by a square post of side-length $a_{\text {sq }}$.

The efficacy of this latter conversion formula is demonstrated by Fig. 6, which contains the CST-generated modal dispersion traces of a SIW that consisted of square pins and compared alongside those of the otherwise identical SIW structure, but composed of circular posts, with the trace markers bearing those corresponding pin shapes. The common SIW parameters are: width $2 b=10 \mathrm{~mm}$, height $h=5 \mathrm{~mm}$, period $d_{x}=0.8 \mathrm{~mm}$, and $\varepsilon_{\text {cen }}=\varepsilon_{\text {left }}=\varepsilon_{\text {right }}=2.2 \varepsilon_{0}$. As for the square pins, $d=t=0.4 \mathrm{~mm}$, giving rise to an equivalent circular pin diameter of $d_{\text {circ }}=0.46863 \mathrm{~mm}$ according to the latter formula. Clearly seen in Fig. 6, the overlap between the traces of both pin shapes is virtually perfect, thereby establishing confidence in this conversion formula and its ability to bridge the investigations of both circular and square pins, thereby justifying the latter.

\section{Mode-Matching With Ordinary Solid-Walled RECTANGULAR WAVEGUIDE}

Among the standout features of the herein presented modal formulation for analyzing SIWs is its ability to treat more complex and practical scenarios beyond just the SIW structure itself, such as waveguide junctions and discontinuities. This section demonstrates just how this is achieved through a particular example of an ordinary homogeneous waveguide connected to a SIW using the mode-matching technique along with the moment method entailing waveguide Green's functions.

Consider in Fig. 7 the schematic of the interconnection between an ordinary solid-walled rectangular waveguide (input section no. 1) homogeneously filled with a medium of relative permittivity $\varepsilon_{\text {rel }}^{\text {in }}$ and an output SIW section (no. 2) immersed within a uniform dielectric host of relative permittivity $\varepsilon_{\mathrm{rel}}^{\mathrm{SIW}}$ separated at $z=z_{1}$. The cross-sectional width and height of

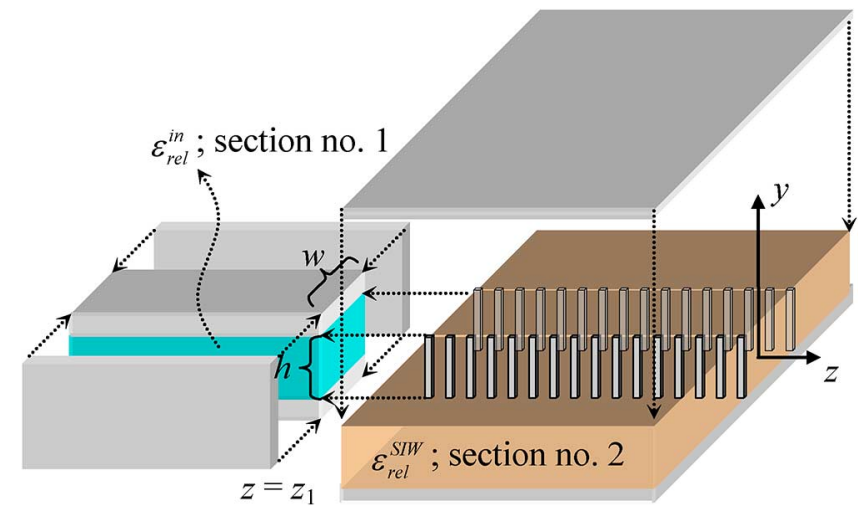

Fig. 7. Ordinary solid-walled input rectangular waveguide connected to an output SIW of the same waveguiding cross section (width $w=2 b$ by height $h$ ) and within dielectric host of relative permittivity $\varepsilon_{\mathrm{rel}}^{\mathrm{SIW}}$

the waveguiding regions of both sections are $w$ and $h$, respectively. Upon invocation of the PEC equivalence theorem [34] thereby entailing equivalent magnetic aperture currents that are expanded into modal basis electric field functions of the input section and using the Green's functions for shorted-end waveguides, the continuity of the tangential magnetic field across the aperture interface at $z=z_{1}$ separating the two sections is enforced, resulting in the following governing equation:

$$
\begin{aligned}
& A_{m_{i}}^{(1)} \vec{h}_{t_{m_{i}}}^{(1)}\left(x_{o}, y_{o}\right) e^{-\gamma_{z_{m_{i}}}^{(1)}\left(z_{1}-z_{s 1}\right)}-\sum_{m=1}^{N_{1}} A_{m}^{(1)} \vec{h}_{t_{m}}^{(1)}\left(x_{o}, y_{o}\right) \\
& =\sum_{p}^{N_{2}} \vec{h}_{t_{p}}^{(2)}\left(x_{o}, y_{o}\right) \sum_{m=1}^{N_{1}} A_{m}^{(1)} C_{1_{m} 2_{p}}, \\
& \quad \text { for }\left(x_{o}, y_{o}\right) \in A_{1} \text { throughout } \\
& C_{1_{m} 2_{p}} \\
& =\int_{y_{s}=-\frac{h}{2} x_{s}=-\frac{w}{2}}^{\frac{h}{2}}\left\{\left[\vec{e}_{t_{m}}^{(1)}\left(x_{s}, y_{s}\right) \times \vec{h}_{t_{p}}^{(2)}\left(x_{s}, y_{s}\right)\right] \cdot \hat{z}\right\} d x_{s} d y_{s}
\end{aligned}
$$

the latter being the modal coupling coefficient between mode $m$ of section no. 1 and mode $p$ of section no. $2 . A_{m_{i}}^{(1)}$ constitutes the known amplitude coefficient of the $+z$ traveling incident $\left(m_{i}\right)$ th modal field coming into the input section (labeled no. 1 , thus the bracketed superscript), whereas $A_{m}^{(1)}$ symbolizes the unknown modal amplitude coefficient of the $m$ th modal basis function that expands the PEC equivalent aperture current. The $h_{t}$ symbols represent the orthonormalized transverse (with $x$ and $y$ components only) modal magnetic field functions of the associated subscripted modal index and of the section indexed by their bracketed superscripts. $\gamma_{z}$ signifies the modal propagation constant (along the guide axis in the $z$-direction) for the mode denoted by its subscripted modal index. $z_{s 1}$ in the exponent of the first term of (24) is the location along the $z$-direction of the excitation source within section no. 1, from which the incident modal field at zero-phase emerges.

Taking $\int_{y_{o}=-h / 2} \int_{x_{o}=-w / 2}\left[\vec{e}_{t_{u}}^{(1)}\left(x_{o}, y_{o}\right) \times \_\cdot \hat{z}\right] d x_{o} d y_{o}$ throughout (24) for $u=1,2, \ldots, N_{1}$ (constituting the 

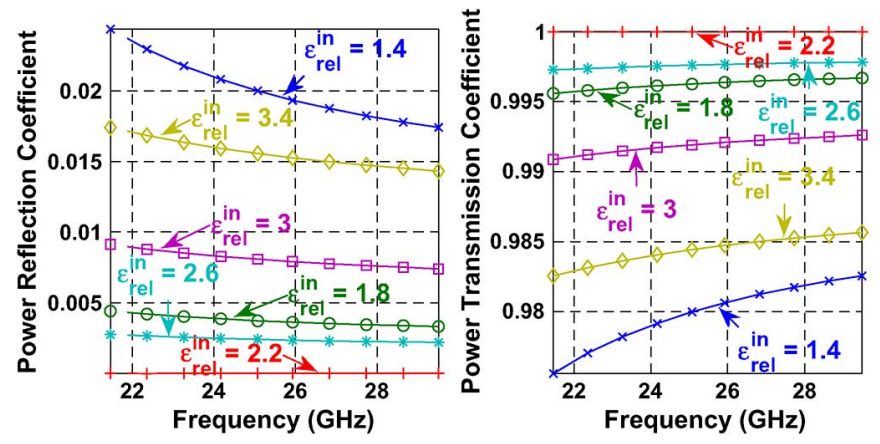

Fig. 8. Variation with frequency of power reflection and transmission coefficients of ordinary solid-walled input rectangular waveguide connected to an output SIW of the same waveguiding cross section $(w=2 b=10 \mathrm{~mm}$, $h=5 \mathrm{~mm}$ ) with pins geometry: $d=1 \mathrm{~mm}, t=0.4 \mathrm{~mm}$, and within dielectric host of relative permittivity $\varepsilon_{\mathrm{rel}}^{\mathrm{SIW}}=2.2$, for various relative permittivities $\varepsilon_{\mathrm{rel}}^{\text {in }}$ homogeneously filling conventional input section, as annotated.

weighting integral operation, $u$ thus being the weighting index) and applying the property of modal orthonormality, we obtain

$A_{u}^{(1)}+\sum_{m=1}^{N_{1}} A_{m}^{(1)} \sum_{p=1}^{N_{2}} C_{1_{m} 2_{p}} C_{1_{u} 2_{p}}=\delta_{u, m_{i}} A_{m_{i}}^{(1)} e^{-\gamma_{z_{m}}^{(1)}\left(z_{1}-z_{s 1}\right)}$

for $u=1,2, \ldots N_{1}$, generating $N_{1}$ equations and thus establishing a solvable system of linear equations for acquiring the unknown modal expansion coefficients $A_{m}^{(1)}$.

For $w=2 b=10 \mathrm{~mm}, h=5 \mathrm{~mm}$, and $\varepsilon_{\text {rel }}^{\mathrm{SIW}}=2.2$, computed results of the reflection and transmission coefficients plotted against frequency for various values of $\varepsilon_{\text {rel }}^{\text {in }}$ are given in Fig. 8. It is observed that the matching between both sections gets improved as the permittivities of their filling materials draw closer to each other, but gets degraded when $\varepsilon_{\mathrm{rel}}^{\text {in }}$ deviates from the value of $\varepsilon_{\text {rel }}^{\mathrm{SIW}}=2.2$, which is as expected.

\section{HARD SIDEWALL SIW}

As mentioned earlier in Section III, the dielectric sidewall loaded hard rectangular waveguide [27] may also be treated under the SIW topology using the present formulation, resulting in the so-called substrate integrated hard sidewall rectangular waveguide, or simply, the hard SIW. Existing analytic and modal methods entailing the expansion of cylindrical wave functions do not lend themselves in the best manner to the treatment of simple slab-type inhomogeneities within the SIW structure with flat surfaces that are parallel with the principal planes of the Cartesian system, such as the case of the hard SIW.

With reference to Fig. 2, Fig. 9 depicts the hard SIW, which comprises the usual SIW, but with a rectangular block of the waveguiding region removed (may generally be replaced by another dielectric slab without restricting the treatment method), resulting in a central portion that consists of a central vacuum domain flanked by two side dielectric regimes representative of the dielectric sidewall loading of conventional hard rectangular waveguides. The various annotated $z$-levels, layer indices, and the coordinate system in the figure correspond to the configuration of Fig. 2.

The following set of parameters shall be investigated: central width $2 b=20 \mathrm{~mm}$, height $h=5 \mathrm{~mm}$, relative permittivity

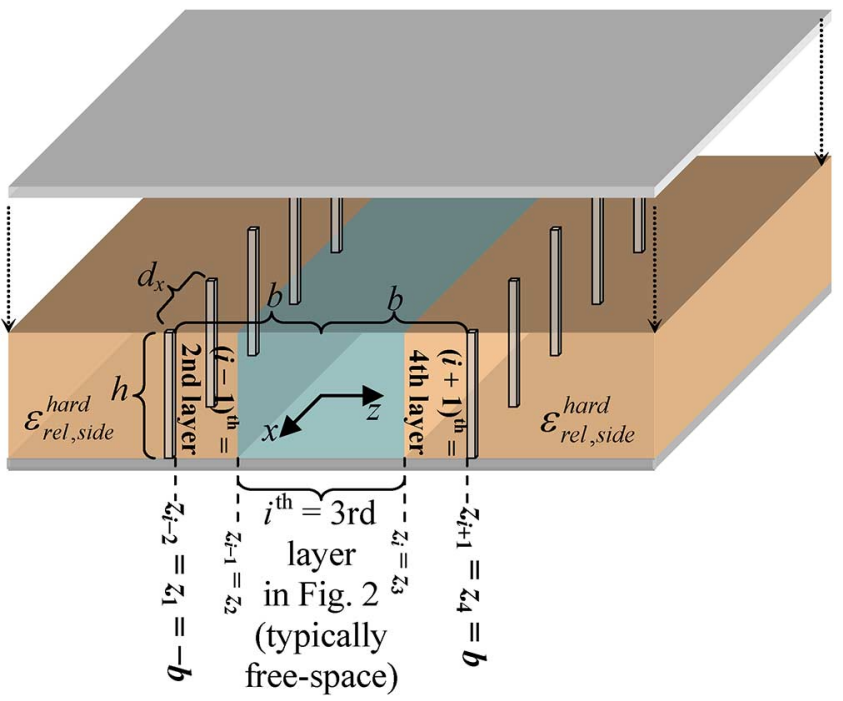

Fig. 9. Schematic of substrate integrated hard sidewall rectangular waveguide. The various annotated $z$-levels, layer indices, and the coordinate system correspond to the configuration of Fig. 2.

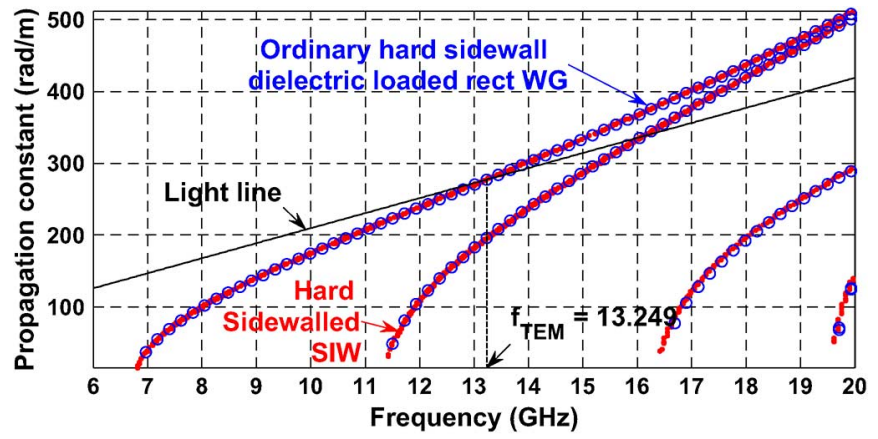

Fig. 10. Dispersion of hard side-walled SIW (fine dots) and its conventional counterpart (circles). For both, width $2 b=20 \mathrm{~mm}$, height $h=5 \mathrm{~mm}$, relative permittivity of identical side dielectric slabs: $\varepsilon_{\mathrm{rel}, \mathrm{side}}^{\mathrm{hard}}=3$, equal sidewall thickness: $z_{2}-z_{1}=z_{4}-z_{3}=d_{\text {hard }}=4 \mathrm{~mm}, f_{\mathrm{TEM}}=13.249 \mathrm{GHz}$. For hard SIW, period $d_{x}=13 \mathrm{~mm}$, pins diameter $d=t=3.883668 \mathrm{~mm}$.

of identical side dielectric slabs: $\varepsilon_{\text {rel,side }}^{\text {hard }}=3$, equal sidewall thickness, $z_{2}-z_{1}=z_{4}-z_{3}=d_{\text {hard }}=4 \mathrm{~mm}$ (refer to Fig. 9 for the $z$ levels), with an associated hard TEM frequency $f_{\text {TEM }}$ of $13.249 \mathrm{GHz}$, obtained from the formula [27]

$$
f_{\mathrm{TEM}}=\left[4 d_{\text {hard }} \sqrt{\mu_{0} \varepsilon_{0}\left(\varepsilon_{\text {rel }, \text { side }}^{\text {hard }}-1\right)}\right]^{-1} .
$$

For an array of square pins, the period is $d_{x}=13 \mathrm{~mm}$ and the diameter is $d=t=3.883668 \mathrm{~mm}$. With these parameters laid out, Fig. 10 conveys the modal dispersion traces of the hard SIW along with those of its conventional counterpart. The emulation by the former with side conducting grilles of the latter encased within solid walls is found to be remarkable. The modal trace of the dominant mode is also seen to intersect the light line at a point that indeed corresponds to $f_{\mathrm{TEM}}$.

The distribution of the $E_{y}$ field component over the central waveguiding region of the hard sidewalled SIW is also mesh plotted at several frequencies about $f_{\text {TEM }}=13.249 \mathrm{GHz}$ in Fig. 11, namely, at (a) $0.83 f_{\mathrm{TEM}}$, (b) $0.909 f_{\mathrm{TEM}}$, (c) $1.004 f_{\mathrm{TEM}}$, and (d) $1.135 f_{\mathrm{TEM}}$. Indeed, the same phenomena 


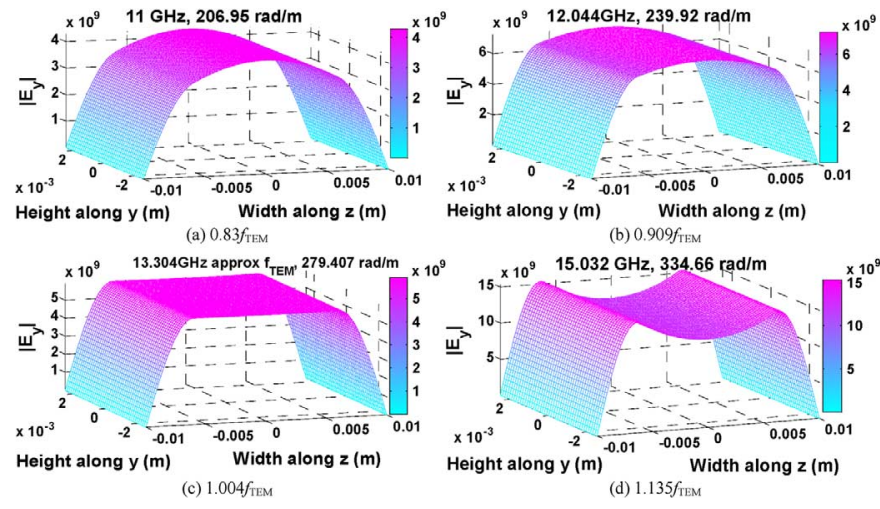

Fig. 11. Field distribution of $E_{y}$ component over central waveguiding region of hard SIW with $f_{\text {TEM }}=13.249 \mathrm{GHz}$ at: (a) $11 \mathrm{GHz}, k_{x}=206.95 \mathrm{rad} / \mathrm{m}$, (b) $12.044 \mathrm{GHz}, k_{x}=239.92 \mathrm{rad} / \mathrm{m}$, (c) $13.304 \mathrm{GHz}, k_{x}=279.407 \mathrm{rad} / \mathrm{m}$, and (d) $15.032 \mathrm{GHz}, k_{x}=334.66 \mathrm{rad} / \mathrm{m}$.

[27] as the ordinary hard sidewalled rectangular waveguide are recovered by the hard SIW, as in, for frequencies below the TEM frequency (the first two showcased frequencies), the field patterns bear sinusoidal variations along the width (z-direction) comprising two space waves bouncing off the sidewalls, whereas for frequencies above $f_{\text {TEM }}$, two sets of surface waves propagate along both side slabs, joining up at the middle of the structure, as Fig. 11(d) shows. Hence, $f_{\text {TEM }}$ serves as the cutoff for surface waves, and at a frequency very close to $f_{\mathrm{TEM}}$, as is for Fig. 11(c), uniform field distribution tantamount to the quasi-TEM hard condition is achieved.

\section{DIELECTRIC LOSSES}

The effects of dielectric losses in the host slab of the SIW can also be treated by the formulated technique. This is achieved through the replacement of the pure real permittivity of the formerly lossless dielectric slab with a complex one of the form

$$
\varepsilon_{\text {lossy }}=\varepsilon^{\prime}-j \varepsilon^{\prime \prime}=\varepsilon^{\prime}(1-j \tan \delta)
$$

with $\tan \delta$ being the loss tangent of the dissipative material, consequently rendering all associated quantities complex, such as the wave impedances of the various lossy regions and the spectral wavenumber components of all Floquet harmonics. Particularly, the axial propagation constant is now represented by $k_{x}=\beta_{x}-j \alpha_{x}$, where $\beta_{x}$ and $\alpha_{x}$ are, respectively, the phase and attenuation constants. By sweeping through a 2-D range of $\beta_{x}$ and $\alpha_{x}$ values over which the solution for $\bar{V}$ in (22) of the entire SIW structure at each coordinate location $\left(\beta_{x}, \alpha_{x}\right)$ is repeatedly carried out as detailed in Sections III-VI, all of which being subjected to a certain predesignated fixed (resonant) frequency $f^{\text {res }}$, the absolute of the sum of the solved amplitude coefficients contained in $\bar{V}$ may be mesh plotted against $\beta_{x}$ and $\alpha_{x}$. The coordinates $\left(\beta_{x}^{\text {res }}, \alpha_{x}^{\text {res }}\right)$ at which a sharp peak occurs in this aforementioned plot then pertain to the detected modal resonance at that associated resonant frequency $f^{\text {res }}$. Repeating this numerical resonance search procedure for other values of $f^{\text {res }}$ consequently permits the separate plots of $\beta_{x}^{\text {res }}$ and $\alpha_{x}^{\text {res }}$ versus $f^{\text {res }}$.

Carrying out the foregoing procedure for $\varepsilon_{\mathrm{cen}}=\varepsilon_{\text {left }}=$ $\varepsilon_{\text {right }}=2.2(1-j 0.016) \varepsilon_{0}$, the mesh plots of the aforesaid ab-
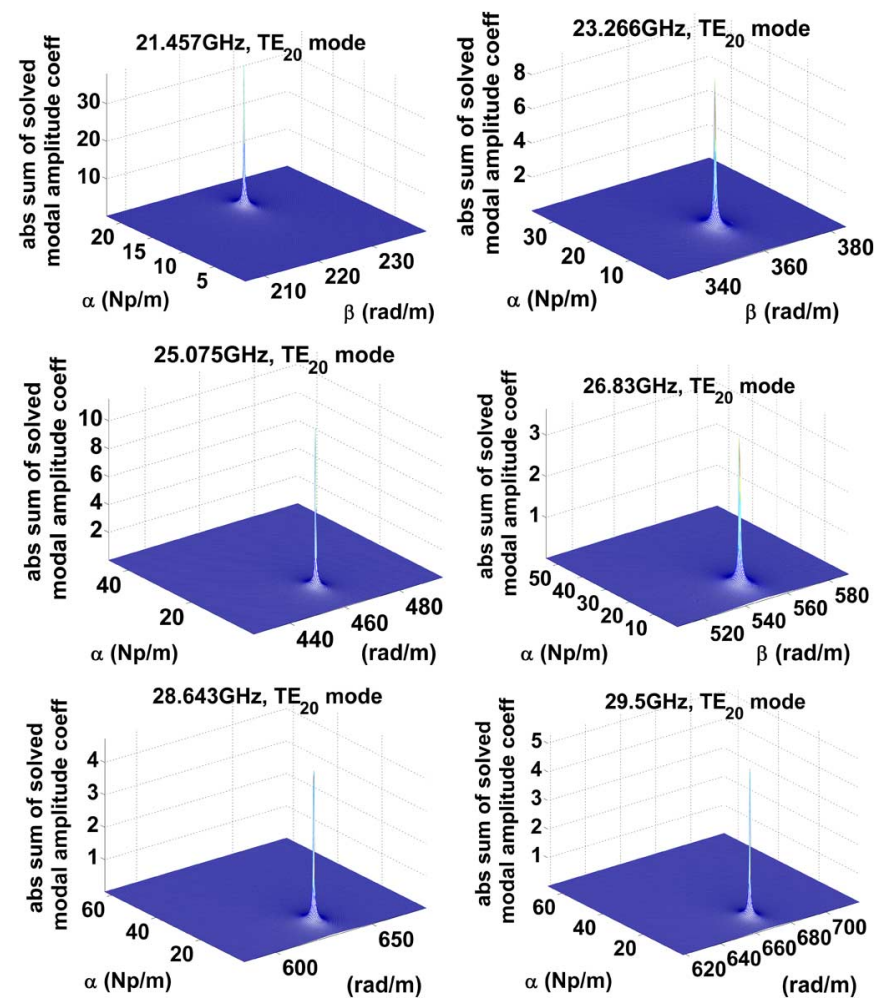

Fig. 12. Mesh plots of absolute sum of solved amplitude coefficients of $\bar{V}$ versus search space over $\beta_{x}$ and $\alpha_{x}$, each for a certain frequency as indicated, all for the $\mathrm{TE}_{20}$ mode, and all for $b=5 \mathrm{~mm}, h=5 \mathrm{~mm}, d=0.2 b=1 \mathrm{~mm}$, $d_{x}=0.8 d=0.8 \mathrm{~mm}, a=d_{x} / 2=0.4 \mathrm{~mm}, \mu_{\mathrm{cen}}=\mu_{\mathrm{left}}=\mu_{\mathrm{right}}=\mu_{0}$, and $\varepsilon_{\text {cen }}=\varepsilon_{\text {left }}=\varepsilon_{\text {right }}=2.2(1-j 0.016) \varepsilon_{0}$.

solute of the sum of the solved modal amplitudes in $\bar{V}$ against $\beta_{x}$ and $\alpha_{x}$ are displayed in Fig. 12 for various frequencies, as indicated in each subplot, all for the second most dominant $\mathrm{TE}_{20}$ mode, arbitrarily selected for illustration (equally effective for the $\mathrm{TE}_{10}$ mode). With reference to Fig. 1, the other parameters are as follows: $b=5 \mathrm{~mm}, h=5 \mathrm{~mm}, d=0.2 b=1 \mathrm{~mm}$, $d_{x}=0.8 d=0.8 \mathrm{~mm}, a=d_{x} / 2=0.4 \mathrm{~mm}$, and $\mu_{\text {cen }}=$ $\mu_{\text {left }}=\mu_{\text {right }}=\mu_{0}$. Indeed, sharp peaks have been detected, as clearly portrayed.

The graph of the dielectric-loss-attributed attenuation constant $\alpha_{x}$ versus frequency associated with the preceding resonance-peak plots for the $\mathrm{TE}_{20}$ mode and $\tan \delta=0.016$ as well as for several other loss tangents $(0.008,0.012,0.02$, and 0.024$)$ are given in Fig. 13(b), along with the corresponding ones of the dominant $\mathrm{TE}_{10}$ mode in Fig. 13(a).

It is observed that the decay escalates with loss tangent, as expected. Higher losses are sustained by the higher order $\mathrm{TE}_{20}$ mode. Attenuation of the dominant mode gets aggravated with rising frequency, whereas that of the $\mathrm{TE}_{20}$ mode falls as frequency increases.

\section{SiDE LEAKAGE}

The leakage through the side apertures created by the gaps between adjacent posts shall now be investigated. Prior works on this issue include [35] and [36]. Let $i$ denote the unit-cell index of the SIW structure. For propagation along the $+x$-direction, the modal powers entering and leaving the $i$ th cell through the cross-sectional input and output aperture ports at $x=i d_{x}-a / 2$ 


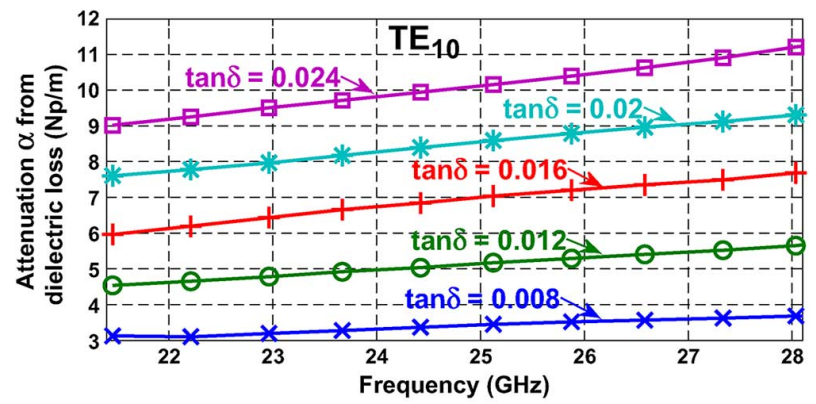

(a)

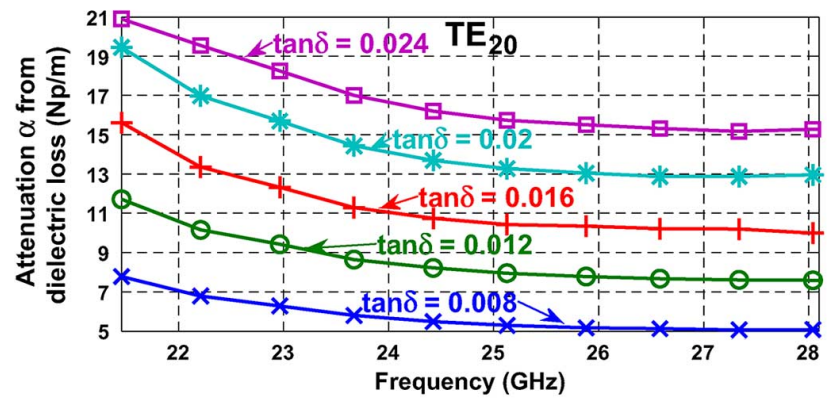

(b)

Fig. 13. Attenuation by dielectric loss versus frequency for: (a) $\mathrm{TE}_{10}$ mode and (b) $\mathrm{TE}_{20}$ mode for various loss tangents: $0.008,0.012,0.016,0.02$, and 0.024 . Universal real parts of $\varepsilon_{\mathrm{rel}}^{\mathrm{cen}}=\varepsilon_{\mathrm{rel}}^{\text {left }}=\varepsilon_{\mathrm{rel}}^{\mathrm{right}}=2.2$ and common width $2 b=10 \mathrm{~mm}$, height $h=5 \mathrm{~mm}, d=0.2 b=1 \mathrm{~mm}, d_{x}=0.8 d=0.8 \mathrm{~mm}$, and aperture gap $a=d_{x} / 2=0.4 \mathrm{~mm}$. Decay escalates with loss tangent, as expected. Higher losses sustained by higher order $\mathrm{TE}_{20}$ mode. Attenuation of dominant mode aggravates with rising frequency, whereas that of $\mathrm{TE}_{20}$ mode falls as frequency increases.

and $i d_{x}+a / 2$ are then represented by $P_{i}^{\text {in }}$ and $P_{i}^{\text {out }}$, all respectively. Subsequently, the amplitude coefficients of the orthonormalized modal field functions at the aforementioned input and output ports of the $i$ th cell are given by $A_{i}^{\text {in }}$ and $A_{i}^{\text {out }}$, being the square roots of twice their respective associated powers. Also let $P_{i}^{\text {leak }}$ be the leakage powers that seep through both side apertures of the $i$ th cell, and $N_{\text {cells }}^{1 m}=\left\lfloor 1 / d_{x}\right\rfloor$ symbolize the total number of full unit-cells within $1 \mathrm{~m}$ of propagation length. Starting off from the known modal amplitude coefficient $A_{1}^{\text {in }}$ of the very first cell assumed to be centered at $x=0$ marking the beginning of the propagation before any leakage loss is incurred, the following recursive relations may be written:

$$
\begin{aligned}
A_{i}^{\text {out }}= & \sqrt{2 P_{i}^{\text {out }}} \\
P_{i}^{\text {out }}= & P_{i}^{\text {in }}-P_{i}^{\text {leak }} ; P_{i}^{\text {in }}=P_{i-1}^{\text {out }}, \quad \text { for } i=2,3, \ldots, N_{\text {cells }}^{1 m} \\
P_{1}^{\text {in }}= & 0.5\left|A_{1}^{\text {in }}\right|^{2} \\
P_{i}^{\text {leak }}= & \left(\frac{A_{i}^{\text {in }}}{A_{1}^{\text {in }}}\right)^{2} \operatorname{Re} \int_{x=i d_{x}-\frac{a}{2}}^{x=i d_{x}+\frac{a}{2}} \int_{y=-\frac{h}{2}}^{y=\frac{h}{2}} \\
& \times\left(\vec{E}_{\text {total }(z=-b)}^{(\ell=2) \text { cen }} \times\left[\vec{H}_{\text {total }(z=-b)}^{(\ell=2) \text { cen }}\right]^{*} \cdot(-\hat{z}) \cdots\right. \\
& \left.\quad+\vec{E}_{\text {total }(z=b)}^{(\ell=4) \text { cen }} \times\left[\vec{H}_{\text {total }(z=b)}^{(\ell=4) \text { cen }}\right]^{*} \cdot \hat{z}\right) d x d y \\
A_{i}^{\text {in }}= & \sqrt{2 P_{i}^{\text {in }}}=A_{i-1}^{\text {out }}=\sqrt{2 P_{i-1}^{\text {out }}}
\end{aligned}
$$

where the modal field functions within the surface integral of the power density vectors for acquiring $P_{i}^{\text {leak }}$ are in their known orthonormalized forms (acquired from the present formulation), with the subscript total denoting that all relevant magnetic current sources responsible for the fields are taken into account. The coefficient $\left(A_{i}^{\mathrm{in}} / A_{1}^{\mathrm{in}}\right)^{2}$ prefixing $P_{i}^{\text {leak }}$ is the so-called decay factor upon the stage of the $i$ th cell. Describing these relations in words, with $A_{1}^{\text {in }}$ and thus $P_{1}^{\text {in }}=0.5\left|A_{1}^{\text {in }}\right|^{2}$ as well as the associated $P_{1}^{\text {leak }}$ with the unit decay factor being known right from the start, $P_{1}^{\text {out }}$ is thus initialized. This latter is then assigned as $P_{2}^{\text {in }}$, leading to the knowledge of $A_{2}^{\text {in }}=\sqrt{2 P_{2}^{\text {in }}}$, and thus also the decay factor for $P_{2}^{\text {leak }}$, which can then be evaluated. These $P_{2}^{\text {in }}$ and $P_{2}^{\text {leak }}$ then generate $P_{2}^{\text {out }}$, which, in turn, spawns the output power through the next (third) cell aperture further down the propagation path, so on and so forth, up until the final one: $P_{N_{\text {cells }}^{1 m} \text { out }}^{\text {out }}$, thus also yielding the associated amplitude coefficient $A_{N_{\text {cells }}^{1 m} \text { out }}^{\text {outs }}=\sqrt{2 P_{N_{\text {cells }}^{1 m}}^{\text {out }}}$ of the modal field function at the furthest output aperture $1 \mathrm{~m}$ away from the input. The ultimate side-leakage attenuation constant is then stated as

$$
\alpha_{\text {leak }}=-\ln \left(\frac{A_{N_{\text {cells }}^{1 m}}^{\text {out }}}{A_{1}^{\text {in }}}\right)(\mathrm{Np} / \mathrm{m}) .
$$

In the discussion of the upcoming numerical results, the following common fixed parameters are first stated: SIW width $2 b=10 \mathrm{~mm}$, height $h=1.5 \mathrm{~mm}, \mu_{\mathrm{cen}}=\mu_{\text {left }}=\mu_{\text {right }}=\mu_{0}$, and $\varepsilon_{\text {cen }}=\varepsilon_{\text {left }}=\varepsilon_{\text {right }}=\varepsilon_{0}$. With these laid out, two varying parameters shall be investigated: 1) the period $d_{x}$ as a fraction of the width $2 b$, i.e., $d_{x} /(2 b)$ and 2$)$ the square-pin diameter $d=t$ as a fraction of the period $d_{x}$, i.e., $t / d_{x}$. For a fixed frequency of $22.573 \mathrm{GHz}$ throughout, Fig. 14 presents the variation of the side-leakage attenuation, $\alpha_{\text {leak }}$, with these two aforementioned parameters, each in a separate graph, within which the various traces pertain to different values of the other parameter. Starting with Fig. 14(a), it is observed that the loss attributed to side leakage gets diminished as the pins occupy an increasing fraction of the unit cell, which is intuitively satisfying. On the other hand, the leakage gets more severe with increasing separation distance between adjacent pins, as Fig. 14(b) indicates, also being an expected result.

The variation of $\alpha_{\text {leak }}$ with frequency is also conveyed in Fig. 15 just for two selected $d_{x} /(2 b)$ cases of 0.3 and 0.4 .

\section{CONDUCTOR LOSSES}

Losses arising from finite conductivities of the metallic side posts as well as the upper (ceiling) and lower (floor) conducting sheets of the SIW can also be treated by the present modal analysis. To obtain the attenuation constant due to conductor losses, the same concept as that for the side-leakage losses in (29) of Section XIII reapplies, but just with the exception of replacing $P_{i}^{\text {leak }}$ there with the following [37]:

$$
P_{i}^{\text {cond }}=\frac{R_{s}}{2} \iint_{S_{i}^{\text {cond }}}\left|\vec{H}_{t}\right|^{2} d s, \quad \text { where } R_{s}=\sqrt{\frac{\pi f \mu_{\text {cond }}}{\sigma}}
$$

being the power lost to the imperfect metals within the $i$ th cell with $S_{i}^{\text {cond }}$ denoting the conducting surfaces inside that cell, which comprises the floor and ceiling of the central waveguiding region as well as the gap between adjacent posts, plus 


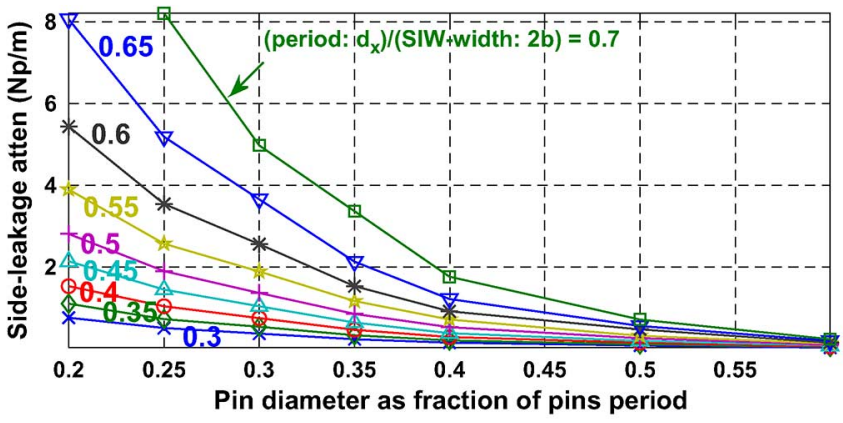

(a)

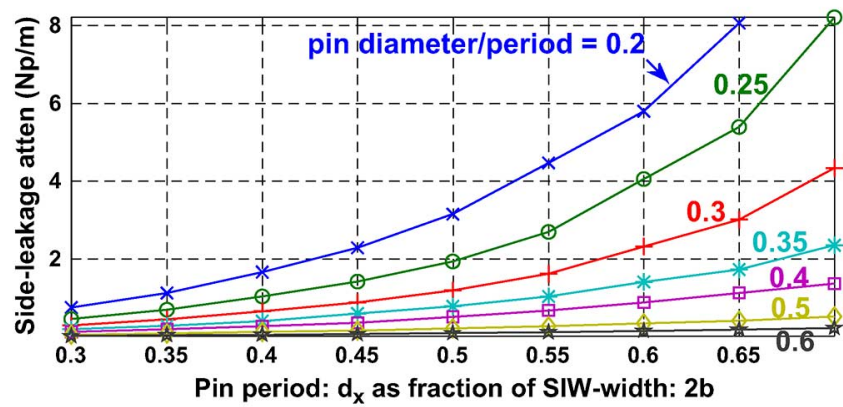

(b)

Fig. 14. Variation of side-leakage attenuation $\alpha_{\text {leak }}$ with: (a) pins diameter as fraction of pins period for various period-to-width ratios and (b) pins period $d_{x}$ as fraction of central width $(2 b)$ for various pins diameter to period ratios, all at $22.573 \mathrm{GHz}$. SIW center width $2 b=10 \mathrm{~mm}$, height $h=1.5 \mathrm{~mm}$, and vacuum throughout (besides pins and upper and lower metal sheets).

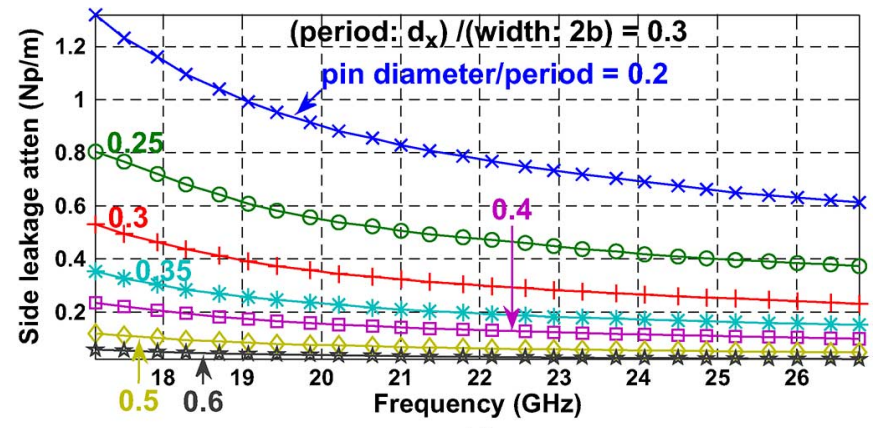

(a)

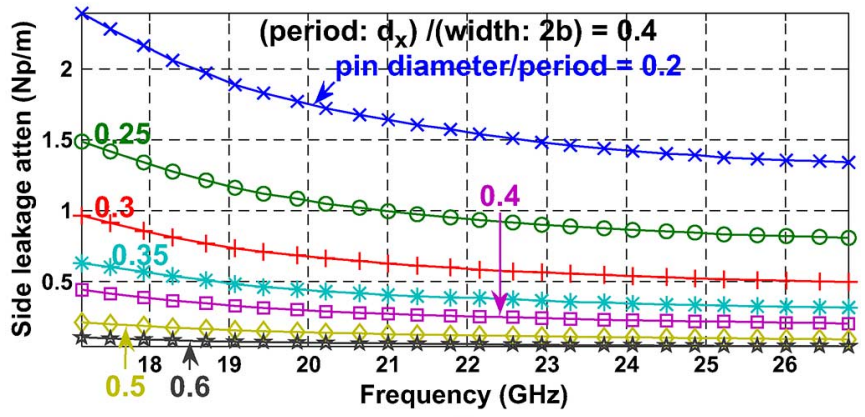

(b)

Fig. 15. Variation of side-leakage attenuation $\alpha_{\text {leak }}$ with frequency for various pins diameter to period ratios (each pertaining to a trace), for period-to-width ratio equals to: (a) 0.3 and (b) 0.4 . SIW width $2 b=10 \mathrm{~mm}$, height $h=1.5 \mathrm{~mm}$, and vacuum throughout (besides pins and metal sheets).

the surfaces of the pins, all within that $i$ th cell, and $H_{t}$ being the tangential magnetic field component over those aforementioned metal surfaces. $R_{s}$ represents the surface resistance of

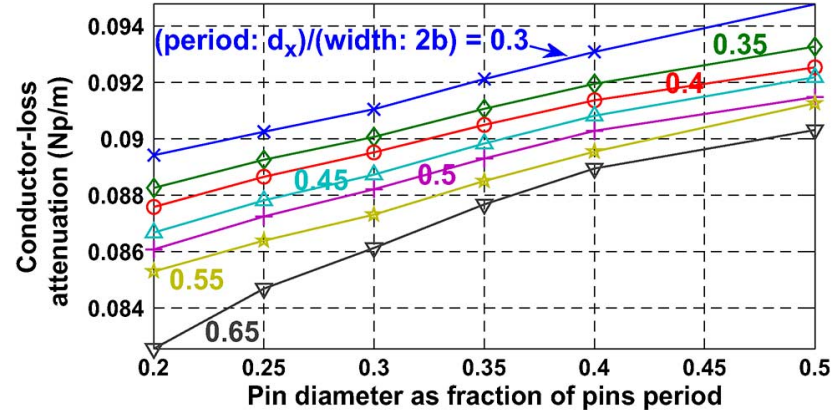

(a)

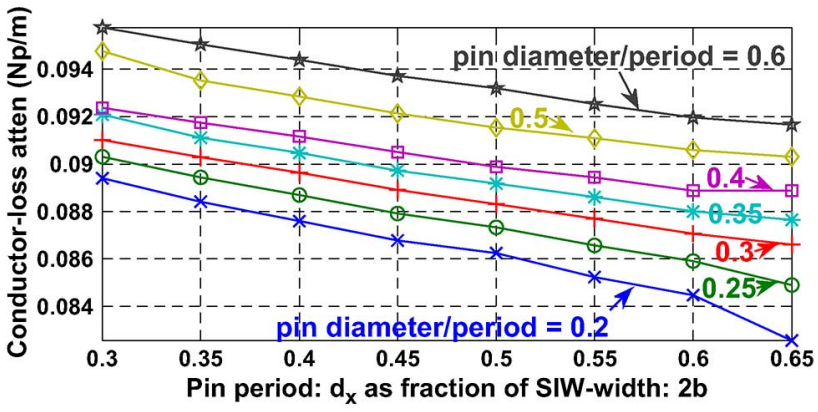

(b)

Fig. 16. Variation of conductor-loss attenuation $\alpha_{\text {cond }}$ with: (a) pins diameter as fraction of pins period for various period-to-width ratios and (b) pins period $d_{x}$ as fraction of central width $(2 b)$ for various pins diameter to period ratios, all at $22.573 \mathrm{GHz}$. Metal is copper with $\sigma=5.8 \times 10^{7} \mathrm{~S} / \mathrm{m}$ and $\mu_{\text {cond }}=\mu_{0}$. SIW center width $2 b=10 \mathrm{~mm}$, height $h=1.5 \mathrm{~mm}$, and vacuum throughout (besides pins and upper and lower metal sheets).

the conductors with $\mu_{\text {cond }}$ and $\sigma$ signifying their permeability and conductivity, respectively.

Consequently, the same mathematical form as of its counterpart version of $\alpha_{\text {leak }}$ in (30) for expressing the conductor-loss attributed attenuation constant, $\alpha_{\text {cond }}$, is stated as

$$
\alpha_{\text {cond }}=-\left.\ln \left(\frac{A_{N_{\text {cell }}^{\text {out }}}^{\text {out }}}{A_{1}^{\text {in }}}\right)\right|_{\text {cond }}(\mathrm{Np} / \mathrm{m})
$$

the subscript cond on the right-hand side incorporated to expressly indicate that the associated terms pertain to the present case of conductor losses, having been acquired using $P_{i}^{\text {cond }}$ of (31) instead of $P_{i}^{\text {leak }}$ in (29).

In the same plotting format as Fig. 14 for leakage losses and using copper as the lossy metal with $\alpha=5.8 \times 10^{7} \mathrm{~S} / \mathrm{m}$ and $\mu_{\text {cond }}=\mu_{0}$, Fig. 16 provides the graphs of $\alpha_{\text {cond }}$ against those same two parameters: $d_{x} /(2 b)$ and $t / d_{x}$ in Fig. 16(a) and (b), respectively. As seen, the trends in variation of $\alpha_{\text {cond }}$ with these two parameters are opposite from those of $\alpha_{\text {leak }}$. Specifically, the conductor losses now instead climbs with expanding pin size relative to the available maximum space provided by the unit cell, but falls as the pins get further apart from each other, both being intuitively satisfying phenomena as well. These countertrends between $\alpha_{\text {leak }}$ and $\alpha_{\text {cond }}$ point to the existence of an optimal condition at which the total attenuation constant, stated as $\alpha_{\text {total }}=\alpha_{\text {leak }}+\alpha_{\text {cond }}$ expressive of the combined effects of leakage and conductor losses, is minimal. This notion shall be explored in Section XV. 


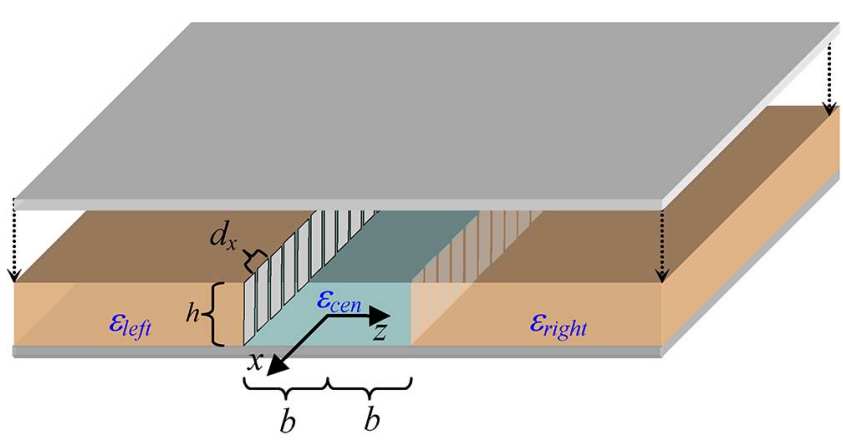

Fig. 17. Sidewall strip-grated SIW with the regular rows of metallic posts replaced by thin strip gratings.

\section{RePlacement OF PINS BY THIN STRIP GRATING}

Extending the notion of rectangular pins by flattening them into thin metallic strips, a modified form of the SIW as schematized in Fig. 17 may be conceived. It becomes a structure that is even lighter in weight, but yet preserves the economy and fabrication ease through cheap printed-circuit and etching techniques long known for cost-effectively realizing strip gratings. Moreover, with printed strips being less invasive on the substrates than via-hole drilling, this variant type of SIW is potentially stronger structurally, especially where the via-holes are punched close together. This section thus explores the prospects of this alternative version of the SIW with near weightless strip-grated sidewalls and whose substrates are not subjected to the stresses from drilling.

While slitting the already adequately lightweight thin-metallaced dielectric sheets might initially appear redundant, it will be demonstrated in this section, in connection with the last sentence of Section XIV, that doing so judiciously yields an optimally low overall attenuation $\alpha_{\text {total }}=\alpha_{\text {leak }}+\alpha_{\text {cond }}$, arising from the combined effects of leakage and conductor losses. This then exemplifies yet another positive and relevant attribute of the present modal analysis of SIW entailing rectangular pins, flattening in one dimension of which become thin metallic strips.

For the upcoming computed results, the following parameters are kept unvaried: width $2 b=10 \mathrm{~mm}$, height $h=1.5 \mathrm{~mm}$, strip thickness $d=0.2 \mathrm{~mm}$, and period $d_{x}=6 \mathrm{~mm}, \varepsilon_{\text {cen }}=\varepsilon_{0}$, $\varepsilon_{\text {left }}=\varepsilon_{\text {right }}=2.2 \varepsilon_{0}$ with $\sigma=5.8 \times 10^{7} \mathrm{~S} / \mathrm{m}$ and $\mu_{\text {cond }}=$ $\mu_{0}$ (copper), leaving just a single remaining varying parameter: $a / d_{x}$, being the aperture (slit) width to period ratio.

The graph of $\alpha_{\text {total }}$ versus $a / d_{x}$ is presented in Fig. 18 with the inset plot showing the corresponding results for the two constituent attenuation constants: $\alpha_{\text {leak }}$ and $\alpha_{\text {cond }}$. As conjectured at the end of Section XIV, a minimum total attenuation constant is indeed existent (at around $a / d_{x}=0.12$ for this case), being a consequence of the converse responses to variations in the period and diameter of the pins exuded by those two avenues of losses. Expressly, the leakage is (of course) low at small $a / d_{x}$, whereas losses to the imperfect copper parts are maximal (also as expected since the side strips are thick). But as $a / d_{x}$ rises, $\alpha_{\text {cond }}$ falls (expectedly since the amount of metal decreases) at a mild steady pace, whereas sure enough, $\alpha_{\text {leak }}$ climbs since the gap between posts widens, increasing only slightly initially for

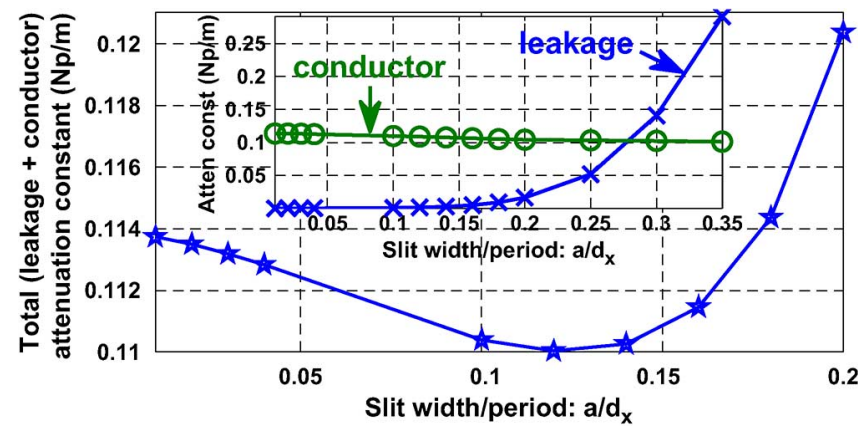

Fig. 18. Total (leakage plus conductor loss) attenuation constant $\alpha_{\text {total }}$ versus slit-width to period ratio: $a / d_{x}$ for sidewall strip-grated SIW (of preceding figure). Width $2 b=10 \mathrm{~mm}$, height $h=1.5 \mathrm{~mm}$, strip thickness $d=0.2 \mathrm{~mm}$, period $d_{x}=6 \mathrm{~mm}, \varepsilon_{\text {cen }}=\varepsilon_{0}, \varepsilon_{\text {left }}=\varepsilon_{\text {right }}=2.2 \varepsilon_{0}$, and $\sigma=5.8 \times 10^{7} \mathrm{~S} / \mathrm{m}$ and $\mu_{\text {cond }}=\mu_{0}$ (copper). Inset graph shows variation of the two constituent attenuation constants $\alpha_{\text {leak }}$ and $\alpha_{\text {cond }}$.

low $a / d_{x}$ levels, but then grows with escalating rate for larger ratios.

\section{CONCLUSIONS}

A rigorous full-wave modal analysis of a SIW has been presented. Based on the method of moments, Green's functions for rectangular cavities and planar multilayer structures are used, those for the latter being in the spectral domain. The Floquet theorem is also thus employed. Concepts behind the numerical search for eigenmodal resonances entailing repeated solutions are also described. Dispersion diagrams in terms of both propagation phase and attenuation constants generated by this treatment approach agree well with those acquired from the commercial software solver CST. Using a known conversion formula relating the geometries of circular pins to those of square ones initially derived for a 2-D lattice of pins, simulations in the present context of SIWs have indeed demonstrated the virtual equivalence between square and circular pins in terms of the modal dispersion characteristics. As such, studies into both pin shapes are bridged, rendering the adoption of either one or the other equally relevant.

The capabilities of the proposed modal formulation in managing scenarios with complexities beyond just the SIW alone have also been exhibited. Particularly, the presence of junctions and discontinuities via the specific example of a regular waveguide connected to a SIW, and material discontinuities within the structure through the showcase of the so-called hard sidewalled SIW.

Three avenues of losses were also investigated using the present technique, namely, dielectric, side-leakage, and imperfect metal losses. It is found that the latter two forms of decay display opposite trends from each other in response to variations in the period of the pins and their diametric size. This gives rise to the existence of an optimal gap separation relative to the unit cell size.

By replacing the conventional volumetric posts with thin facial strip gratings etched onto the side surfaces of the dielectric slabs, the weight of the SIW can be brought down even lower without compromising the low cost and convenience due to printed circuit board (PCB) and etching technologies that are 
just as cheap and accessible. By exhibiting the best tradeoff between side leakage and conductor losses, the prospects of this modified form of SIW with virtually weightless sidewall strip gratings have also been established.

\section{APPENDIX A}

\section{EXPLICIT ORTHONORMALIZED RECTANGULAR WAVEGUIDE MODAL FIELD FUNCTIONS}

The transverse waveguide modal field functions entailed throughout this paper must be in their orthonormalized forms, as required by the dyadic cavity Green's functions. For a coordinate-origin centered rectangular waveguide with width and height along $x$ and $y$ being $a$ and $h$, respectively, and homogeneously filled with a medium of parameters $\left(\mu_{\text {cav }}, \varepsilon_{\text {cav }}\right)$, the following are stated:

$$
\begin{aligned}
& e_{t_{p q \mathrm{TE}}}^{\overrightarrow{\mathrm{cav}}^{\mathrm{a}}}(x, y) \\
& =\left\{\begin{array}{l}
\hat{x} \sqrt{\frac{2 Z_{0 q}^{\mathrm{TE}}}{a h}} S_{y}, \quad p=0, q \neq 0 \\
-\hat{y} \sqrt{\frac{2 Z_{p 0}^{\mathrm{TE}}}{a h}} S_{x}, \quad p \neq 0, q=0 \\
\frac{2}{k_{c_{p q}}^{\text {cav }}} \sqrt{\frac{Z_{p q}^{\mathrm{TE}}}{a h}}\left(\hat{x} k_{y_{q}} C_{x} S_{y}-\hat{y} k_{x_{p}} S_{x} C_{y}\right), \quad p \neq 0, q \neq 0
\end{array}\right.
\end{aligned}
$$$$
\vec{h}_{t_{p q \mathrm{TE}}}^{\mathrm{cav}}(x, y)
$$$$
=\left\{\begin{array}{l}
\hat{y} \sqrt{\frac{2 Y_{0 q}^{\mathrm{TE}}}{a h}}\left[S_{y}\right], \quad p=0, q \neq 0 \\
\hat{x} \sqrt{\frac{2 Y_{p 0}^{\mathrm{TE}}}{a h}}\left[S_{x}\right], \quad p \neq 0, q=0 \\
\frac{2}{k_{c p q}^{\text {cav }}} \sqrt{\frac{Y_{p q}^{\mathrm{TE}}}{a h}}\left(\hat{x} k_{x_{p}} S_{x} C_{y}+\hat{y} k_{y_{q}} C_{x} S_{y}\right), \quad p \neq 0, q \neq 0
\end{array}\right.
$$$$
\vec{e}_{t_{p q T M} \text { cav }(x, y)}
$$$$
=-\frac{2}{k_{c_{p q}}^{\mathrm{cav}}} \sqrt{\frac{Z_{p q}^{\mathrm{TM}}}{a h}}\left(\hat{x} k_{x_{p}} C_{x} S_{y}+\hat{y} k_{y_{q}} S_{x} C_{y}\right)
$$$$
\vec{h}_{t_{p q T M}}^{\mathrm{cav}}(x, y)
$$$$
=\frac{2}{k_{c_{p q}}^{\mathrm{cav}}} \sqrt{\frac{Y_{p q}^{\mathrm{TM}}}{a h}}\left(\hat{x} k_{y_{q}} S_{x} C_{y}-\hat{y} k_{x_{p}} C_{x} S_{y}\right)
$$

where

$$
\begin{aligned}
k_{x_{p}} & =\frac{p \pi}{a} \\
k_{y_{q}} & =\frac{q \pi}{h} \\
k_{c_{p q}}^{\mathrm{cav}} & =\sqrt{k_{x_{p}}^{2}+k_{y_{q}}^{2}} \\
\left\{\begin{array}{l}
C_{x} \\
S_{x}
\end{array}\right\} & =\left\{\begin{array}{l}
\cos \\
\sin
\end{array}\right\}\left(k_{x_{p}} x+\frac{p \pi}{2}\right) \\
\left\{\begin{array}{l}
C_{y} \\
S_{y}
\end{array}\right\} & =\left\{\begin{array}{l}
\cos \\
\sin
\end{array}\right\}\left(k_{y_{q}} y+\frac{q \pi}{2}\right) \\
Z_{p q}^{\mathrm{TE}} & =j \omega \mu_{\mathrm{cav}} / \gamma_{z_{p q}}^{\mathrm{cav}}=\left(Y_{p q}^{\mathrm{TE}}\right)^{-1} \\
Z_{p q}^{\mathrm{TM}} & =\gamma_{z_{p q}}^{\mathrm{cav}} / j \omega \varepsilon_{\mathrm{cav}}=\left(Y_{p q}^{\mathrm{TM}}\right)^{-1} \\
\gamma_{z_{p q}}^{\mathrm{cav}} & =\sqrt{\left(k_{c_{p q}}^{\mathrm{cav}}\right)^{2}-k_{\mathrm{cav}}^{2}} \\
k_{\mathrm{cav}} & =\omega \sqrt{\mu_{\mathrm{cav}} \varepsilon_{\mathrm{cav}}} .
\end{aligned}
$$

In this way, the following orthonormality property holds:

$$
\begin{gathered}
\int_{y=-h / 2}^{y=h / 2} \int_{x=-a / 2}^{x=a / 2}\left(\vec{e}_{t_{p q r}}^{\mathrm{cav}} \times \vec{h}_{t_{u v w}}^{\mathrm{cav}}\right) \cdot \hat{z} d x d y \\
=\delta_{p q r, u v w}= \begin{cases}1, & \text { if } p=u, q=v, r \equiv w \\
0, & \text { otherwise. }\end{cases} \\
\text { APPENDIX B }
\end{gathered}
$$

For the $i$ th layer homogeneously filled with material $\left(\mu_{i}, \varepsilon_{i}\right)$ bounded by $z_{i-1}$ and $z_{i}$ with wavenumber $k_{i}=2 \pi f \sqrt{ }\left(\mu_{i} \varepsilon_{i}\right)$, impedance $\eta_{i}=\sqrt{ }_{\text {ex }}\left(\mu_{i} / \varepsilon_{i}\right)$, and the presence of spectral excitation sources: $\tilde{\tilde{\Psi}}_{w}^{z_{i}^{*}}$ ( $\Psi$ may be $J$ or $M$, and $w$ is $x, y$ or $z$ ) located at level $z_{i}^{\mathrm{ex}}$, the fields within this $i$ th layer are given by the following:

$$
\begin{aligned}
& {\left[\begin{array}{c}
\tilde{\tilde{E}}_{x}^{(i)} \\
\tilde{\tilde{E}}_{y}^{(i)} \\
\tilde{\tilde{E}}_{z}^{(i)}
\end{array}\right]=\frac{1}{2}\left\{\left[\begin{array}{cc}
R_{b-}^{\alpha} & R_{b+}^{\alpha} \\
S_{b-}^{\alpha} & S_{b+}^{\alpha} \\
T_{b-}^{\alpha} & T_{b+}^{\alpha}
\end{array}\right]\left[\begin{array}{c}
\chi^{-} \\
\chi^{+}
\end{array}\right]\right.} \\
& \left.+\left[\begin{array}{c}
\tilde{\tilde{E}}_{x\left(z_{<} z_{i}^{\mathrm{ex}}\right)}^{(i) \mathrm{DPR}} \\
\tilde{\tilde{E}}_{y\left(z_{<} \geq z_{i}^{\mathrm{ex}}\right)}^{(i) \mathrm{DPR}} \\
\left.\tilde{\tilde{E}}_{z\left(z_{<}(i) \mathrm{DPR}\right.}^{\mathrm{ex}}\right)
\end{array}\right] e^{-j k_{z_{i}}\left|z-z_{i}^{\mathrm{ex}}\right|}\right\} \\
& {\left[\begin{array}{c}
\tilde{\tilde{H}}_{x}^{(i)} \\
\tilde{\tilde{H}}_{y}^{(i)} \\
\tilde{\tilde{H}}_{z}^{(i)}
\end{array}\right]=\frac{1}{2}\left\{-\left[\begin{array}{cc}
R_{b-}^{\beta} & R_{b+}^{\beta} \\
S_{b-}^{\beta} & S_{b+}^{\beta} \\
T_{b-}^{\beta} & T_{b+}^{\beta}
\end{array}\right]\left[\begin{array}{l}
\chi^{-} \\
\chi^{+}
\end{array}\right]\right.} \\
& \left.+\left[\begin{array}{c}
\tilde{\tilde{H}}_{x\left(z_{<} z_{i}^{\mathrm{ex}}\right)}^{(i) \mathrm{DPR}} \\
\tilde{\tilde{H}}_{y\left(z^{>} z_{i}^{\text {ex }}\right)}^{(i) \mathrm{DPR}} \\
\tilde{\tilde{H}}_{z\left(z_{<} z_{i}^{\text {ex }}\right)}^{(i) \mathrm{DPR}}
\end{array}\right] e^{-j k_{z_{i}}\left|z-z_{i}^{\text {ex }}\right|}\right\}
\end{aligned}
$$

whereby $\chi^{ \pm}=\exp \left[ \pm j k_{z_{i}}\left(z-z_{\left\{\begin{array}{c}i \\ i-1\end{array}\right\}}\right)\right]$ and

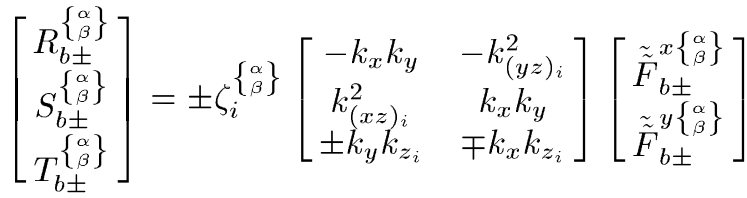

$$
\begin{aligned}
& +\frac{\left\{\begin{array}{l}
+1 \\
-1
\end{array}\right\}}{k_{z_{i}}}\left[\begin{array}{cc}
k_{z_{i}} & 0 \\
0 & k_{z_{i}} \\
\pm k_{x} & \pm k_{y}
\end{array}\right]\left[\begin{array}{c}
\tilde{\tilde{G}}_{b \pm}^{x\{}\left\{\begin{array}{c}
\alpha \\
\beta
\end{array}\right\} \\
\tilde{\tilde{G}}_{b \pm}^{y}\left\{\begin{array}{l}
\alpha \\
\beta
\end{array}\right\}
\end{array}\right]
\end{aligned}
$$

in which $\zeta_{i}^{\left\{\begin{array}{c}\alpha \\ \beta\end{array}\right\}}=\eta_{i}^{ \pm 1}\left(k_{i} k_{z_{i}}\right)^{-1}$,

$$
\begin{aligned}
{\left[\begin{array}{l}
\tilde{\tilde{F}}_{b \pm}^{\{x, y\}(\gamma)} \\
\tilde{\tilde{G}}_{b \pm}^{\{x, y\}(\gamma)}
\end{array}\right]=} & {\left[\begin{array}{ll}
\delta_{\gamma \alpha} & \delta_{\gamma \beta} \\
\delta_{\gamma \beta} & \delta_{\gamma \alpha}
\end{array}\right]\left[\begin{array}{c}
\tilde{\tilde{H}}_{b \pm}^{\{x, y\}} \\
\tilde{\tilde{E}}_{b \pm}^{\{x, y\}}
\end{array}\right] } \\
b \pm= & i-\frac{1}{2} \pm \frac{1}{2} \\
k_{(u z)_{i}}^{2}= & k_{i}^{2}-k_{v}^{2}=k_{u}^{2}+k_{z_{i}}^{2} \\
& u=x ; \quad v=y \text { or } u=y ; \quad v=x
\end{aligned}
$$


where $\delta_{u v}$ is the Kronecker delta, being unity when its two subscript indices are identical and zero otherwise, and with

$$
\begin{aligned}
& \tilde{\tilde{\psi}}_{x\left(z_{<}^{>} z_{i}^{\mathrm{ex}}\right)}^{(i) \operatorname{DPR}}=\zeta_{i}^{\psi}\left[-k_{(y z)_{i}}^{2} \tilde{\tilde{K}}_{x_{i}}^{\psi}+k_{x} k_{y} \tilde{\tilde{K}}_{y_{i}}^{\psi} \pm k_{x} k_{z_{i}} \tilde{\tilde{K}}_{z_{i}}^{\psi}\right]+\cdots \\
& +\left(\delta_{\psi E}-\delta_{\psi H}\right)\left(\mp \tilde{\tilde{L}}_{y_{i}}^{\psi}+\frac{k_{y}}{k_{z_{i}}} \tilde{\tilde{L}}_{z_{i}}^{\psi}\right) \\
& \tilde{\tilde{\psi}}_{y\left(z_{<} z_{i}^{\text {ex }}\right)}^{(i) \mathrm{DPR}}=\zeta_{i}^{\psi}\left[k_{x} k_{y} \tilde{\tilde{K}}_{x_{i}}^{\psi}-k_{(x z)_{i}}^{2} \tilde{\tilde{K}}_{y_{i}}^{\psi} \pm k_{y} k_{z_{i}} \tilde{\tilde{K}}_{z_{i}}^{\psi}\right]+\cdots \\
& +\left(\delta_{\psi E}-\delta_{\psi H}\right)\left( \pm \tilde{\tilde{L}}_{x_{i}}^{\psi}-\frac{k_{x}}{k_{z_{i}}} \tilde{\tilde{L}}_{z_{i}}^{\psi}\right) \\
& \tilde{\tilde{\psi}}_{z\left(z_{<}^{>} z_{i}^{\text {ex }}\right)}^{(i) \text { DPR }}=\zeta_{i}^{\psi}\left[ \pm k_{x} k_{z_{i}} \tilde{\tilde{K}}_{x_{i}}^{\psi} \pm k_{y} k_{z_{i}} \tilde{\tilde{K}}_{y_{i}}^{\psi}-\left(k_{i}^{2}-k_{z_{i}}^{2}\right) \tilde{\tilde{K}}_{z_{i}}^{\psi}\right] \\
& +\cdots+\frac{\delta_{\psi E}-\delta_{\psi H}}{k_{z_{i}}}\left(-k_{y} \tilde{\tilde{L}}_{x_{i}}^{\psi}+k_{x} \tilde{\tilde{L}}_{y_{i}}^{\psi}\right)
\end{aligned}
$$

where $\zeta_{i}^{\left\{\begin{array}{l}E \\ H\end{array}\right\}}=\eta_{i}^{ \pm 1}\left(k_{i} k_{z_{i}}\right)^{-1}$ and $\psi$ may denote $E$ or $H$, with DPR signifying "direct path radiation" from the excitation sources, and

$$
\left[\begin{array}{c}
\tilde{\tilde{K}}_{w_{i}}^{\psi} \\
\tilde{\tilde{L}}_{w_{i}}^{\psi}
\end{array}\right]=\left[\begin{array}{cc}
\delta_{\psi E} & \delta_{\psi H} \\
\delta_{\psi H} & \delta_{\psi E}
\end{array}\right]\left\{\begin{array}{c}
\tilde{\tilde{J}}_{w}^{z^{\mathrm{ex}}} \\
\tilde{\tilde{M}}_{w}^{z_{i}^{\text {ex }}}
\end{array}\right\}, \quad w=x, y, z .
$$

At this juncture, it is noted that $\tilde{\tilde{\Psi}}_{w}^{z_{i}^{\text {ex }}}$ need not be nonzero in all layers, and for those in which no sources exist, this excitation source term vanishes, and thus the DPR fields in those sourceless layers as well.

The spectral boundary field components, $\tilde{\tilde{E}}_{b \pm}^{\{x, y\}}$ and $\tilde{\tilde{H}}_{b \pm}^{\{x, y\}}$ are acquired from the core routine by the solution of the multilayer structure, details of which are as follows.

Define the following elemental quantities:

$$
\begin{aligned}
& \varepsilon_{ \pm}=\exp \left[ \pm j k_{z_{\left\{\begin{array}{c}
i+1 \\
i
\end{array}\right.}}\left(z_{i}-z_{\left\{\begin{array}{l}
i+1 \\
i-1
\end{array}\right\}}\right)\right]
\end{aligned}
$$

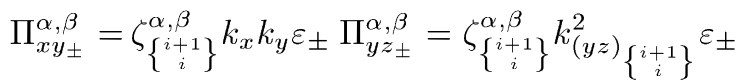

$$
\begin{aligned}
& \Pi_{x z_{ \pm}}^{\alpha, \beta}=\zeta_{\left\{\begin{array}{c}
i+1 \\
i
\end{array}\right\}}^{\alpha, \beta} k_{(x z){ }_{\left\{\begin{array}{c}
i+1 \\
i
\end{array}\right\}}^{2}}^{2} \varepsilon_{ \pm} \\
& \Phi_{y z}^{\alpha, \beta}=\zeta_{i}^{\alpha, \beta} k_{(y z)_{i}}^{2}+\zeta_{i+1}^{\alpha, \beta} k_{(y z)_{i+1}}^{2} \\
& \Phi_{x z}^{\alpha, \beta}=\zeta_{i}^{\alpha, \beta} k_{(x z)_{i}}^{2}+\zeta_{i+1}^{\alpha, \beta} k_{(x z)_{i+1}}^{2} \\
& \Omega^{\alpha, \beta}=k_{x} k_{y}\left(\zeta_{i}^{\alpha, \beta}+\zeta_{i+1}^{\alpha, \beta}\right)
\end{aligned}
$$

in all of which the upper and lower signs of \pm correspond to the upper and lower quantities in the curly braces.

Elemental $2 \times 2$ sub-matrix building blocks are defined as

$$
\begin{aligned}
\bar{\varepsilon}_{ \pm} & =\left[\begin{array}{cc}
\varepsilon_{ \pm} & 0 \\
0 & \varepsilon_{ \pm}
\end{array}\right] \\
\bar{\Pi}_{ \pm}^{\alpha, \beta} & =\left[\begin{array}{cc}
\Pi_{x y \pm}^{\alpha, \beta} & \Pi_{y z_{ \pm}}^{\alpha, \beta} \\
-\Pi_{x z_{ \pm}, \beta}^{\alpha, \beta} & -\Pi_{x y_{ \pm}}^{\alpha, \beta}
\end{array}\right] \\
\bar{\Theta}^{\alpha, \beta} & =\left[\begin{array}{cc}
-\Omega^{\alpha, \beta} & -\Phi_{y z}^{\alpha, \beta} \\
\Phi_{x z}^{\alpha, \beta} & \Omega^{\alpha, \beta}
\end{array}\right]
\end{aligned}
$$

such that the $4 \times 12$ fundamental matrix $\bar{A}_{i}$ is stated as

$$
\bar{A}_{i}=\left[\begin{array}{cccccc}
\bar{\varepsilon}_{-} & \bar{\Pi}_{-}^{\alpha} & \overline{0} & \bar{\Theta}^{\alpha} & -\bar{\varepsilon}_{+} & \bar{\Pi}_{+}^{\alpha} \\
\bar{\Pi}_{-}^{\beta} & -\bar{\varepsilon}_{-} & \bar{\Theta}^{\beta} & \overline{0} & \bar{\Pi}_{+}^{\beta} & \bar{\varepsilon}_{+}
\end{array}\right]_{4 \times 12}
$$

where $\overline{0}$ is a $2 \times 2$ zero matrix, and noting the $i$ subscript attached to $\bar{A}$ to signify that it pertains to a certain $i$ th interface level at $z_{i}$ whose boundary conditions entail the fields in both the $i$ th and $(i+1)$ th layers.

The known excitation $4 \times 1$ sub-column-vector associated with this $\bar{A}_{i}$ is given by $\bar{C}_{i}=\left[\begin{array}{llll}C_{11} & C_{21} & C_{31} & C_{41}\end{array}\right]^{\mathrm{T}}$, where

$$
\begin{aligned}
& C_{11}=-\tilde{\tilde{E}}_{x\left(z>z_{i}^{\mathrm{ex}}\right)}^{(i) \mathrm{DPR}} \chi_{-}^{\mathrm{ex}}+\tilde{\tilde{E}}_{x\left(z<z_{i}^{\mathrm{ex}}\right)}^{(i+1) \mathrm{DPR}} \chi_{+}^{\mathrm{ex}} \\
& C_{21}=-\tilde{\tilde{E}}_{y\left(z>z_{i}^{\mathrm{ex}}\right)}^{(i) \mathrm{DPR}} \chi_{-}^{\mathrm{ex}}+\tilde{\tilde{E}}_{y\left(z<z_{i}^{\mathrm{ex}}\right)}^{(i+1) \mathrm{DPR}} \chi_{+}^{\mathrm{ex}} \\
& C_{31}=-\tilde{\tilde{H}}_{x\left(z>z_{i}^{\mathrm{ex}}\right)}^{(i) \mathrm{DPR}} \chi_{-}^{\mathrm{ex}}+\tilde{\tilde{H}}_{x\left(z<z_{i}^{\mathrm{ex}}\right)}^{(i+1) \mathrm{DPR}} \chi_{+}^{\mathrm{ex}} \\
& C_{41}=-\tilde{\tilde{H}}_{y\left(z>z_{i}^{\mathrm{ex}}\right)}^{(i) \mathrm{DPR}} \chi_{-}^{\mathrm{ex}}+\tilde{\tilde{H}}_{y\left(z<z_{i}^{\mathrm{ex}}\right)}^{(i+1) \mathrm{DPR}} \chi_{+}^{\mathrm{ex}}
\end{aligned}
$$

with

$$
\chi_{ \pm}^{\mathrm{ex}}=\exp \left[ \pm j k_{z_{\left\{\begin{array}{c}
i \\
i+1
\end{array}\right\}}}\left(z_{i}-z_{\left\{\begin{array}{c}
i \\
i+1
\end{array}\right\}}^{\mathrm{ex}}\right)\right] .
$$

Define $\bar{B}_{i}=\left[\begin{array}{cccc}\tilde{\tilde{E}}_{i}^{x} & \tilde{\tilde{E}}_{i}^{y} & \tilde{\tilde{H}}_{i}^{x} & \tilde{\tilde{H}}_{i}^{y}\end{array}\right]^{\mathrm{T}}$ such that the associated $12 \times$ 1 sub-column-vector containing the 12 unknowns is expressed as $\bar{B}=\left[\begin{array}{lll}\bar{B}_{i-1} & \bar{B}_{i} & \bar{B}_{i+1}\end{array}\right]^{\mathrm{T}}$, where the superscript $\mathrm{T}$ denotes nonconjugate transpose.

Subsequently, the sub-matrix equation pertaining to the four boundary conditions at $z_{i}$ for $2<i<N_{b}-1\left(N_{b}\right.$ is the total number of boundaries, being equal to $N_{l}-1$, where $N_{l}$ is the total number of layers) is stated as $\bar{A}_{i} \bar{B}=\bar{C}_{i}$.

The final combined matrix $\bar{M}_{\text {core }}$ is then constructed by placing in it the $4 \times 12$ sub-matrix $\bar{A}$ spanning row indices from $4(i-1)+1$ to $4(i-1)+4$ and column indices from $4(i-2)+1$ to $4(i-2)+12$ for $i=2$ to $N_{b}-1$. In this way, a matrix that is banded about the diagonal is formed.

Upon cutting away the first four columns from $\bar{A}_{i=1}$, the resultant $4 \times 8$ matrix, called $\bar{D}$, being

$$
\bar{D}=\bar{A}_{i=1}(:, 5: 12)=\left[\begin{array}{cccc}
\overline{0} & \bar{\Theta}^{\alpha} & -\bar{\varepsilon}_{+} & \bar{\Pi}_{+}^{\alpha} \\
\bar{\Theta}^{\beta} & \overline{0} & \bar{\Pi}_{+}^{\beta} & \bar{\varepsilon}_{+}
\end{array}\right]_{i=1}
$$

fills the first four rows and first eight columns of $\bar{M}_{\text {core }}$.

Likewise, by removing the last four columns of $\bar{A}_{i=N_{b}}$, the resultant $4 \times 8$ matrix, called $\bar{E}$, being

$$
\bar{E}=\bar{A}_{i=N_{b}}(:, 1: 8)=\left[\begin{array}{cccc}
\bar{\varepsilon}_{-} & \bar{\Pi}_{-}^{\alpha} & \overline{0} & \bar{\Theta}^{\alpha} \\
\bar{\Pi}_{-}^{\beta} & -\bar{\varepsilon}_{-} & \bar{\Theta}^{\beta} & \overline{0}
\end{array}\right]_{i=N_{b}}
$$

fills the last four rows and last eight columns of $\bar{M}_{\text {core }}$, spanning row indices from $4\left(N_{b}-1\right)+1$ to $4\left(N_{b}-1\right)+4$ and column indices from $4\left(N_{b}-2\right)+1$ to $4\left(N_{b}-2\right)+12$.

Ultimately, a $4 N_{b} \times 4 N_{b}$ square matrix $\bar{M}_{\text {core }}$ is constructed. All other unspecified element locations are filled by zeros. 
The final combined $4 N_{b} \times 1$ column vector, $\bar{F}$ containing the unknown spectral boundary field components is stated as

$$
\bar{F}=\left[\begin{array}{llll}
\bar{B}_{i=1} & \bar{B}_{i=2} & \cdots & \bar{B}_{i=N_{b}}
\end{array}\right]^{\mathrm{T}}
$$

and the combined known excitation $4 N_{b} \times 1$ column vector, $\bar{G}$ is expressed by

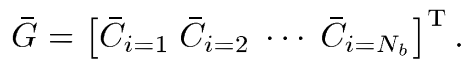

The ultimate matrix equation is then written as

$$
\bar{M}_{\text {core }} \bar{F}=\bar{G}
$$

which may then be solved for $\bar{F}$ via matrix inversion. Doing so yields the spectral boundary field components, $\tilde{\tilde{E}}_{b \pm}^{\{x, y\}}$ and $\tilde{\tilde{H}}_{b \pm}^{\{x, y\}}$ mentioned earlier.

For any spectral excitation source(s), $\tilde{\tilde{\Psi}}_{\sigma}^{z_{\tau}^{\mathrm{ex}}}$ at $z_{\tau}^{\mathrm{ex}}$ in the $\tau$ th layer, with $\sigma$ being $x, y$, or $z$, (B1a) and (B1b) may be perceived as expressions for fields within the $i$ th layer (generally different from $\tau$ th) via the concept of the Green's function, i.e.,

$$
\tilde{\tilde{F}}_{w_{\Psi_{\tau}^{\tau}}}^{(i)}=\tilde{\tilde{G}}_{F_{w}}^{(i)} \bullet \tilde{\tilde{\Psi}}_{\sigma}^{z_{\tau}^{\mathrm{ex}}}
$$

where $F$ is $E$ or $H, w$ may be $x, y$, or $z, \Psi$ is $J$ or $M$, and $\tilde{\tilde{G}}$ is the numerical spectral Green's function for a specific field type and component $F_{w}$.

Bottommost Layer PEC-for Fig. 3(b): When the bottommost layer is PEC, the following changes are made to the matrix (B7).

(a) To the matrix $\bar{M}_{\text {core }}$,

(i) cut out the first two columns of $\bar{M}_{\text {core }}\left(\tilde{\tilde{E}}_{1}^{x}\right.$ and $\tilde{\tilde{E}}_{1}^{y}$ are zero and no longer unknowns);

(ii) cut out the third and fourth rows of $\bar{M}_{\text {core }}$ (no more continuity of the tangential magnetic field across the lowermost PEC boundary interface);

(iii) shift the first two rows of $\bar{M}_{\text {core }}$ down to fill up the void left by the removed third and fourth rows.

(b) To the unknown vector $\bar{F}$ (containing the unknown tangential spectral boundary field components on all $N_{b}$ boundaries): just cut away the first two elements since $\tilde{\tilde{E}}_{1}^{x}=\tilde{\tilde{E}}_{1}^{y}=0$ and are no longer unknowns.

(c) To the known excitation vector $\bar{G}$ : just cut away the third and fourth elements since the third and fourth boundarycondition equations are removed (step (a)(ii) above).

(d) All quantities involving $k_{1}, k_{z 1}$, and $\eta_{1}$ are ignored in the matrix (B7). Specifically, only in the first four row equations will these quantities arise.

Topmost Layer PEC-for Fig. 3(a): When the uppermost $\left(N_{b}+1\right)$ th layer is a PEC, corresponding to a PEC boundary surface at $z=z_{N_{b}}$, the following changes are made to the matrix (B7).

(a) To the matrix $\bar{M}_{\text {core }}$ :

(i) cut out the fourth and third last columns of $\bar{M}_{\text {core }}\left(\tilde{\tilde{E}}_{N_{b}}^{x}\right.$ and $\tilde{\tilde{E}}_{N_{b}}^{y}$ are zero and no longer unknowns); (ii) shift the last two columns to the left to fill up the void created by the removed fourth and third last columns;

(iii) cut out the last two rows of $\bar{M}_{\text {core }}$ (no more continuity of tangential magnetic field across the topmost PEC boundary interface).

(b) To the unknown vector (containing the unknown tangential boundary field components on all $N$ boundaries): just cut off the fourth and third last elements since $\tilde{\tilde{E}}_{N_{b}}=$ $\tilde{\tilde{E}}_{N_{b}}^{y}=0$ and are no longer unknowns.

(c) To the known excitation vector $\bar{G}$ : just cut away the last two elements since the last two boundary-condition equations are removed (step (a)(iii) above).

(d) All quantities involving $k_{N_{b}+1}, k_{z_{N_{b}+1}}$, and $\eta_{N_{b}+1}$ are ignored in matrix (B7). Specifically, only in the last four row equations will these quantities arise.

Both Lowermost and Uppermost Layers PEC-for Fig. 2: When both the bottommost and topmost layers are composed by the PEC, then the sets of changes outlined just above for these two cases are implemented at the same time.

\section{REFERENCES}

[1] J. Hirokawa and M. Ando, "Single-layer feed waveguide consisting of posts for plane TEM wave excitation in parallel plates," IEEE Trans. Antennas Propag., vol. 46, no. 5, pp. 625-630, May 1998.

[2] Y. Cassivi, L. Perregrini, P. Arcioni, M. Bressan, K. Wu, and G. Conciauro, "Dispersion characteristics of substrate integrated rectangular waveguide," IEEE Microw. Wireless Compon. Lett., vol. 12, no. 9, pp. 333-335, Sep. 2002.

[3] F. Xu and K. Wu, "Guided-wave and leakage characteristics of substrate integrated waveguide," IEEE Trans. Microw. Theory Techn., vol. 53 , no. 1, pp. 66-73, Jan. 2005.

[4] S. Germain, D. Deslandes, and K. Wu, "Development of substrate integrated waveguide power dividers," in IEEE Can. Elect. Comput. Eng. Conf., Montreal, QC, Canada, May 2003, pp. 1921-1924.

[5] D. S. Eom, J. Byun, and H. Y. Lee, "Multilayer substrate integrated waveguide four-way out-of-phase power divider," IEEE Trans. Microw. Theory Techn., vol. 57, no. 12, pp. 3469-3476, Dec. 2009.

[6] D. Deslandes and K. Wu, "Single substrate integration technique of planar circuits and waveguide filters," IEEE Trans. Microw. Theory Techn., vol. 51, no. 2, pp. 593-596, Feb. 2003.

[7] W. D'Orazio, K. Wu, and J. Helszajn, "A substrate integrated waveguide degree-2 circulator," IEEE Microw. Wireless Compon. Lett., vol. 14, no. 5, pp. 207-209, May 2004.

[8] W. D'Orazio and K. Wu, "Substrate-integrated-waveguide circulators suitable for millimeter-wave integration," IEEE Trans. Microw. Theory Techn., vol. 54, no. 10, pp. 3675-3680, Oct. 2006.

[9] Q. H. Lai, W. Hong, and Z. Q. Kuai, "Half-mode substrate integrated waveguide transverse slot array," IEEE Trans. Antennas Propag., vol. 57, no. 4, pp. 1064-1072, Apr. 2010.

[10] M. Ohira, A. Miura, and M. Ueba, "60-GHz wideband substrate integrated waveguide slot array using closely spaced elements for planar multisector antenna," IEEE Trans. Antennas Propag., vol. 58, no. 3, pp. 993-998, Mar. 2010.

[11] K. Hashimoto, J. Hirokawa, and M. Ando, "A post-wall waveguide center-feed parallel plate slot array antenna in the millimeter-wave band," IEEE Trans. Antennas Propag., vol. 58, no. 11, pp. 3522-3538, Nov. 2010.

[12] Z. L. Li, X. P. Chen, and K. Wu, "A surface mountable pyramidal horn antenna and transition to substrate integrated waveguide," in Proc. Int. Signals Syst. Electron. Symp., Aug. 2007, pp. 607-610.

[13] H. Wang, D. G. Fang, B. Zhang, and W. Q. Che, "Dielectric loaded substrate integrated waveguide (SIW) H-plane horn antennas," IEEE Trans. Antennas Propag., vol. 58, no. 3, pp. 640-647, Mar. 2010.

[14] B. Pan, Y. Li, G. E. Ponchak, J. Papapolymerou, and M. M. Tentzeris, "A 60-GHz CPW-fed high-gain and broadband integrated horn antenna," IEEE Trans. Antennas Propag., vol. 57, no. 4, pp. 1050-1056, Apr. 2009. 
[15] C. I. Yeh, D. H. Yang, T. H. Liu, J. S. Fu, K. S. Chin, J. C. Cheng, H. C. Chiu, and C. P. Kao, "MMIC compatibility study of SIW H-plane horn antenna," in Proc. Int. Microw. Millimeter-Wave Technol. Conf., May 2010, pp. 933-936.

[16] A. R. Mallahzadeh and S. Esfandiarpour, "Wideband H-plane horn antenna based on ridge substrate integrated waveguide (RSIW)," IEEE Antennas Wireless Propag. Lett., vol. 11, pp. 85-88, 2012.

[17] Z. Hao, W. Hong, J. Chen, X. Chen, and K. Wu, "A novel feeding technique for antipodal linearly tapered slot antenna array," in IEEE MTT-S Int. Microw. Symp., Jun. 2005, pp. 1641-1643.

[18] T. Djerafi and K. Wu, "Corrugated substrate integrated waveguide (SIW) antipodal linearly tapered slot antenna array fed by quasi-triangular power divider," Progr. Electromagn. Res. C, vol. 26, pp. 139-151, 2012.

[19] E. Moldovan, R. G. Bosisio, and K. Wu, "W-band multiport substrateintegrated waveguide circuits," IEEE Trans. Microw. Theory Techn., vol. 54, no. 2, pp. 625-632, Feb. 2006.

[20] I. Wood, D. Dousset, J. Bornemann, and S. Claude, "Linear tapered slot antenna with substrate integrated waveguide feed," in Proc. IEEE AP-S Int. Symp., Honolulu, HI, USA, Jun. 2007, pp. 4761-4764.

[21] D. Dousset, K. Wu, and S. Claude, "Millimetre-wave broadband transition of substrate-integrated waveguide to rectangular waveguide," IET Electron. Lett., vol. 46, no. 24, pp. 1610-1611, Nov. 2010.

[22] Z. Wang, S. Adhikari, D. Dousset, C.-W. Park, and K. Wu, "Substrate integrated waveguide (SIW) power amplifier using CBCPW-to-SIW transition for matching network," in IEEE MTT-S Int. Microw. Symp. Dig., Montreal, QC, Canada, Jun. 2012.

[23] M. Buchta and W. Heinrich, "On the equivalence between cylindrical and rectangular via-holes in electromagnetic modeling," in Proc. 37th Eur. Microw. Conf., Munich, Germany, Oct. 2007, pp. 142-145.

[24] H. Seki, "An alternative representation of electromagnetic fields in a rectangular waveguide with an aperture and its wall," in IEICE Nat. Conv. Rec., Sep. 1984, vol. 1, p. 16.

[25] H. Seki, "Analysis of waveguide directional filters by the moment method," Int. J. Microw. Millimeter-Wave Comput.-Aided Eng., vol. 3, no. 3, pp. 183-191, 1993.

[26] M. Ng Mou Kehn, M. Nannetti, A. Cucini, S. Maci, and P.-S. Kildal, "Analysis of dispersion in dipole-FSS loaded hard rectangular waveguide," IEEE Trans. Antennas Propag., vol. AP-54, no. 8, pp. 2275-2282, Aug. 2006.

[27] M. Ng Mou Kehn and P.-S. Kildal, "Miniaturized rectangular hard waveguides for use in multi-frequency phased arrays," IEEE Trans. Antennas Propag., vol. AP 53, no. 1, pp. 100-109, Jan. 2005.

[28] E. Abaei, E. Mehrshahi, and H. R. Sadreazami, "Analysis of substrate integrated waveguide based on two dimensional multi-port method," in Proc. ICMMT, 2010, pp. 793-796.

[29] E. Abaei, E. Mehrshahi, G. Amendola, E. Arnieri, and A. Shamsafar, "Two dimensional multi-port method for analysis of propagation characteristics of substrate integrated waveguide," Progr. Electromagn. Res. C, vol. 29, pp. 261-273, 2012.
[30] J. Bornemann and F. Taringou, "Mode matching analysis of substrateintegrated waveguide circuits," in Proc. 24th Can. Electr. Comput. Eng. Conf., Niagara Falls, ON, Canada, May 2011, pp. 579-582.

[31] J. Bornemann and F. Taringou, "Substrate-integrated waveguide filter design using mode-matching technique," in 4lst Eur. Microw. Conf., Manchester, U.K., 2011, pp. 1-4.

[32] Z. Kordiboroujeni and J. Bornemann, "Efficient design of substrate integrated waveguide power dividers for antenna feed systems," in Proc. 7th Eur. Antennas Propag. Conf., 2013, pp. 352-356.

[33] G. K. F. Serres, A. J. R. Serres, R. C. S. Freire, C. Fontgalland, and H Baudrand, "Approximation of a rectangular via hole from a cylindrical cross section using the fundamental TM mode," in Proc. SBMO/IEEE MTT-S Int. Microw. Optoelectron. Conf., Rio de Janeiro, Brazil, Aug. 2013, pp. 1-4.

[34] C. A. Balanis, Advanced Engineering Electromagnetics. New York, NY, USA: Wiley, 1989.

[35] M. Bozzi, M. Pasian, L. Perregrini, and K. Wu, "On the losses in substrate-integrated waveguides and cavities," Int. J. Microw. Wireless Technol., vol. 1, no. 5, pp. 395-401, Oct. 2009.

[36] W. Che, D. Wang, K. Deng, and Y. L. Chow, "Leakage and ohmic losses investigation in substrate-integrated waveguide," Radio Sci., vol. 42, 2007, Art. ID RS5005.

[37] D. M. Pozar, Microwave Engineering, 2nd ed. New York, NY, USA: Wiley, 1998.

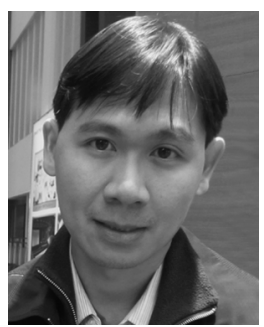

Malcolm Ng Mou Kehn (S'02-M'06-SM'13) received the B.Eng. degree in electrical engineering (with honors) from the National University of Singapore, Singapore, in 2001, and the Licentiate and $\mathrm{Ph} . \mathrm{D}$. degrees in electrical engineering from the Chalmers University of Technology, Göteborg, Sweden, in 2004 and 2005, respectively.

From 2006 to 2008, he was a Postdoctoral Fellow with the Department of Electrical and Computer Engineering, University of Manitoba, Winnipeg, MB, Canada. He then joined Concordia University, Montreal, QC, Canada, for another year of postdoctoral research. In August 2009, he joined the Department of Electrical Engineering, National Chiao Tung University (NCTU), Hsinchu, Taiwan, initially as an Assistant Professor. Since August 2012, he has been an Associate Professor with NCTU. From 2002 to 2006, he was actively involved in research projects funded by the Swedish Defense Research Agency. During Autumn 2004, he spent several months on a research visit with the University of Siena, Siena, Italy. From 2006 to 2009, he was extensively involved with numerous projects supported by Canadian industry and national research bodies. Since August 2009, he has been securing research project grants funded by the National Science Council of Taiwan (now the Ministry of Science and Technology, Taiwan).

Dr. Ng Mou Kehn was the recipient of the Union Radio-Scientifique Internationale (URSI) Young Scientist Award (2007) and the NCTU Meritorious Teaching Award (2013 and 2014). 